\title{
Developing Methods to Circumvent the Conundrum of Chromosomal Rearrangements Occurring in Multiplex Gene Edition
}

Vinciane Borsenberger ${ }^{1, *}$, Christian Croux $^{1}$, Fayza Daboussi ${ }^{1,2}$, Cécile Neuvéglise ${ }^{3}$, Florence Bordes ${ }^{1}$.

${ }^{1}$ TBI, Université de Toulouse, INSA, INRAE, CNRS, Toulouse, France

${ }^{2}$ TWB, 3 rue Ariane, 31520 Ramonville Saint Agne, France

${ }^{3}$ Université Paris-Saclay, INRAE, AgroParisTech, Micalis Institute, 78350, Jouy-en-Josas, Paris, France

* To whom correspondence should be addressed. Tel: +33 (0)5 615594 55; Email:

vinciane.borsenberger@insa-toulouse

Present Address: Cécile Neuvéglise, SPO, INRAE, Montpellier SupAgro, Université Montpellier, 34060

Montpellier, France

\section{Supporting Information}




\section{Table of content}

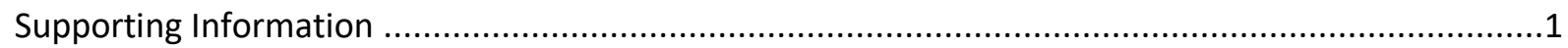

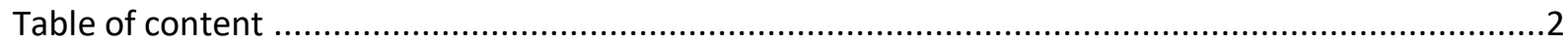

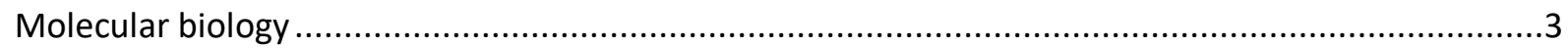

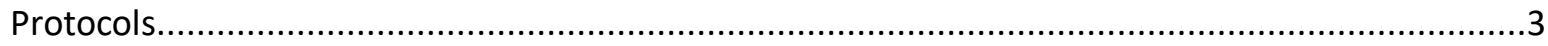

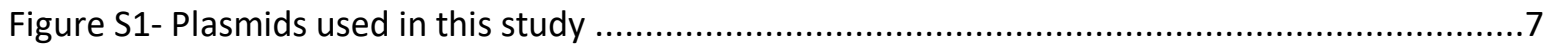

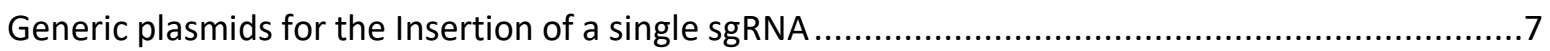

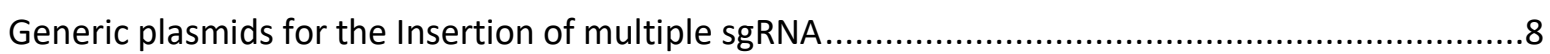

Figure S2 Primer design for multiplex assembly fragments ...................................................10

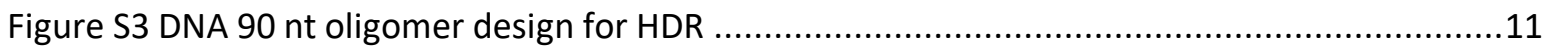

Figure S4 Comparison of transformation efficiencies between single and co-transformations .......12

Figure S5 Toxicity of multiple sgRNA co-expression is associated to DSB formation ......................13

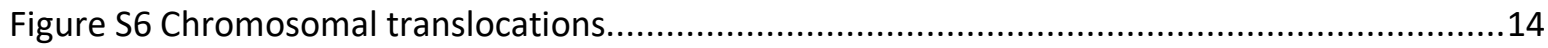

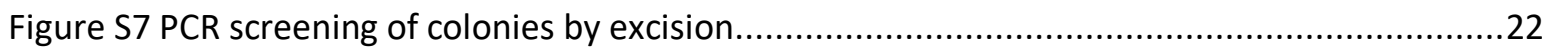

Figure S8-Differential growth of POX disruptants depending on fatty acid complementation ........24

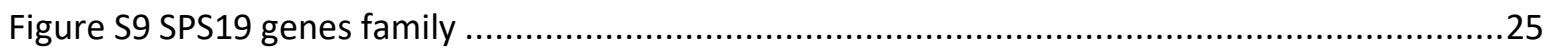

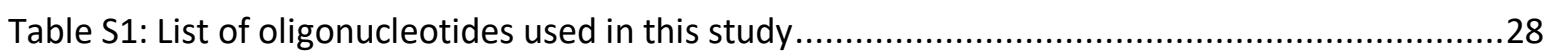

Tables S2 Sequence identity inside $Y$. lipolytica gene families and CRISPR/Cas9 targets .................30

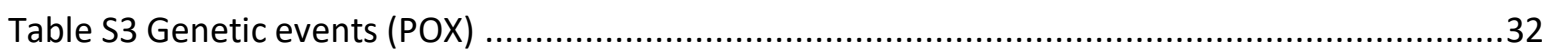

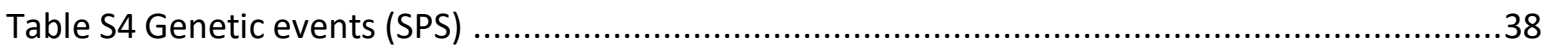

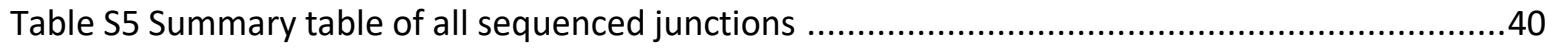

Table S6 Origin of large DNA fragment insertion in SPS disruptants in large insertion events.........41

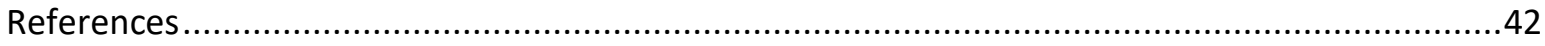




\section{Molecular biology}

\section{Protocols}

\section{General methods for plasmid construction}

Standard molecular biology protocols were applied for basic cloning. DNA concentrations were assessed using NanoDrop technology on an Eon spectrophotometer (Biotek). Plasmid digestions for constructions were typically performed using commercial restriction enzymes (NEB) and gel purified with a gel extraction kit (Sigma). Ligations were typically carried out at $21^{\circ} \mathrm{C}$ for $2 \mathrm{~h}$ with T4 DNA ligase (NEB). PCR reactions were carried out using CloneAmp HiFi PCR Premix (Clontech) in a T120 or a MiniJ cycler (both from Biorad). For construction necessitating an InFusion cloning approach (Clontech), fragments were generated either with restriction digestion followed by gel purification or by PCR amplification followed by spin column purification (PCR purification kit from Macherey Nagel). Escherichia coli strain XL1 blue (Agilent) was used for cloning and plasmid production. For routine cloning, the cells were rendered competent using the Mix \& Go Competent cells kit (Zymo Research) and transformed following the kit manufacturer protocol. Clones were grown in LB media supplemented with ampicillin $(100 \mu \mathrm{g} / \mathrm{mL})$ at $37^{\circ} \mathrm{C}$ and plasmids were subsequently extracted with the Plasmid Miniprep Kit (Qiagen). Plasmid construction of pCg58 and pCg22 can be found in previous work ${ }^{1}$. Backbone plasmids generated for this study were deposited in the Addgene repository (Addgene ID for pCg58: 153304, pCg69: 153305, pg7: 153306, and pg10: 153307).

\section{Removal of SapI restriction sites}

The LEU2 gene inserted in pCg22 was mutated to remove a Sap/ restriction site conditions using CloneAmp HiFi PCR Premix:

Plasmid pCg22

primers

CloneAmp HiFi PCR Premix (2X)

Total reaction volume

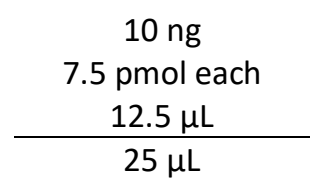

The plasmids were amplified in a PCR cycler with the following parameters:

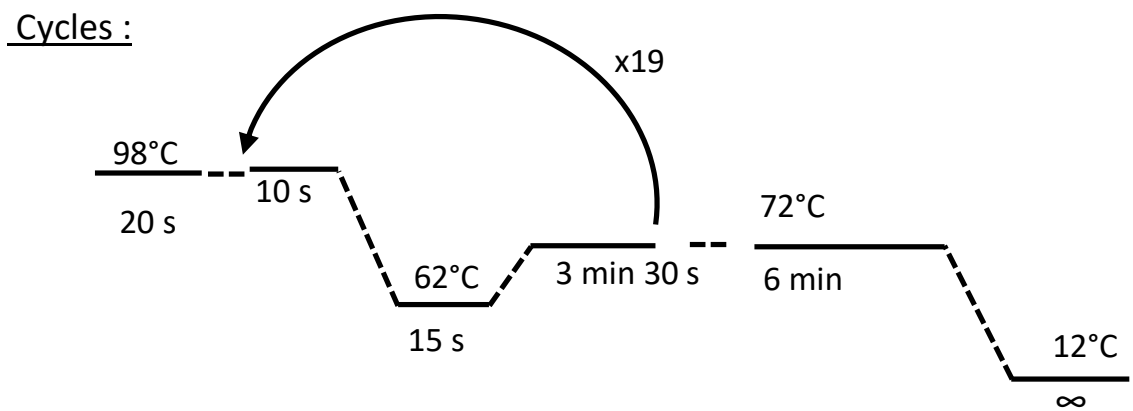

The PCRs reactions were digested with Dpnl, purified and transformed in XL1 blue competent cells.

The LEU2-wo-Sap/ fragment was cloned between AatII and Mlul sites in a similar fashion as described in previous work ${ }^{1}$, eventually yielding $\mathrm{pCg} 57$ generic plasmid, similar to $\mathrm{pCg} 58$, with LEU2 in place of URA3 expression cassette. 


\section{Cas9 cassettes removal from generic plasmids}

The Cas 9 expression cassette were excised from pCg57 and pCg58 by Mlul/Kpnl digestion, the vector band was gel purified and self-ligated using small compatibles oligonucleotides adaptors (see table 1), yielding pg7 and pg8 respectively.

\section{Introduction of an insert with 2 SapI restriction sites enabling the Golden Gate multiplex constructions}

Directed mutagenesis was performed on pg7, pg8 and pCg58 to introduce two Sapl sites at the target location using Muta_Sapl_GG_For and Muta_Sapl_GGBB_rev (see SI table 1), yielding pg10, pg11, and $\mathrm{pCg} 69$ respectively.

Plasmid pg7, pg8, or pCg58 primers

CloneAmp HiFi PCR Premix (2X)

Total reaction volume

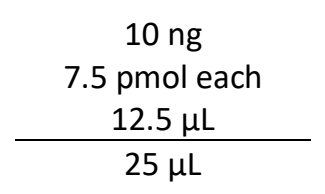

The plasmids were amplified in a PCR cycler with the following parameters:

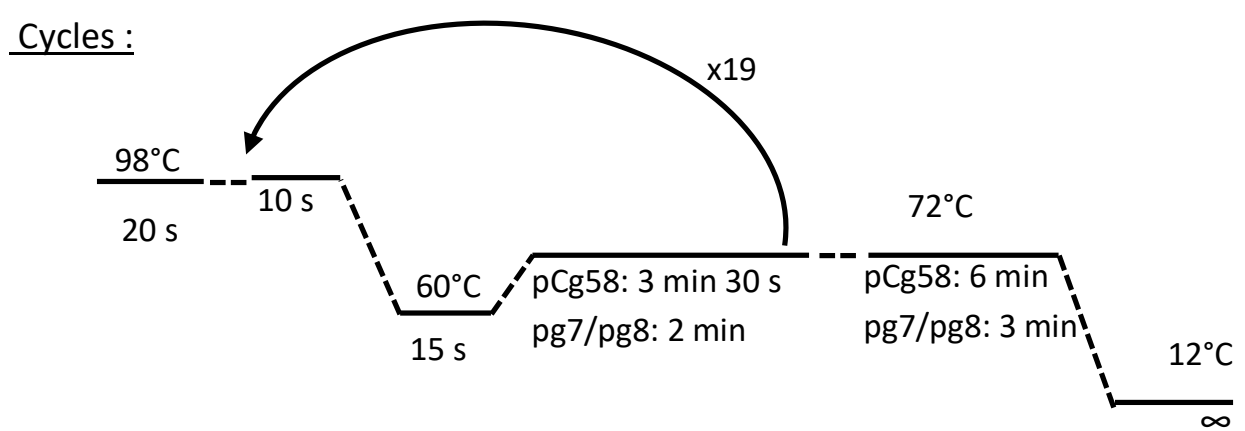

The PCRs reactions were digested with Dpnl, purified and transformed in XL1 blue competent cells.

\section{Insertion of target sequences in generic pCg58 and pg7 plasmids}

The targets were inserted by Golden Gate reaction with Sapl and T4 ligase of ds oligonucleotides (see table S1) in pg7 and pCg58 backbone plasmids, according to the procedure described in Borsenberger et al. ${ }^{1}$

After propagation, plasmids were verified by digestion with Aatll and Sapl restriction enzymes. Correct insertion in pCg58 yields two bands at $636 \mathrm{bp}$ and $9173 \mathrm{bp}$, while WT pCg58 gives 3 bands at $636 \mathrm{bp}, 3228 \mathrm{bp}$ and $5922 \mathrm{bp}$. Conversely, correct target insertion in pg7 yields a single band at 5632 bp, while WT pg7 gives 2 bands at 2381and $3228 \mathrm{bp}$.

Correct clones were confirmed by sequencing with the primer Seq_guide. (See table1). 


\section{Amplicons for Golden gate assembly in multiplex plasmid backbones}

The fragments were amplified from pCg58 or pg 7 plasmids with the inserted targets. See SI Fig S2 to choose the appropriate primer combinations.

Plasmid

$20 \mathrm{ng}$

Primers $(10 \mu \mathrm{M})$

$7.5 \mathrm{pmol}$

CloneAmp HiFi PCR Premix (2X)

$12.5 \mu \mathrm{L}$

Nuclease free water

to $25 \mu \mathrm{L}$

Total reaction volume

$25 \mu \mathrm{L}$

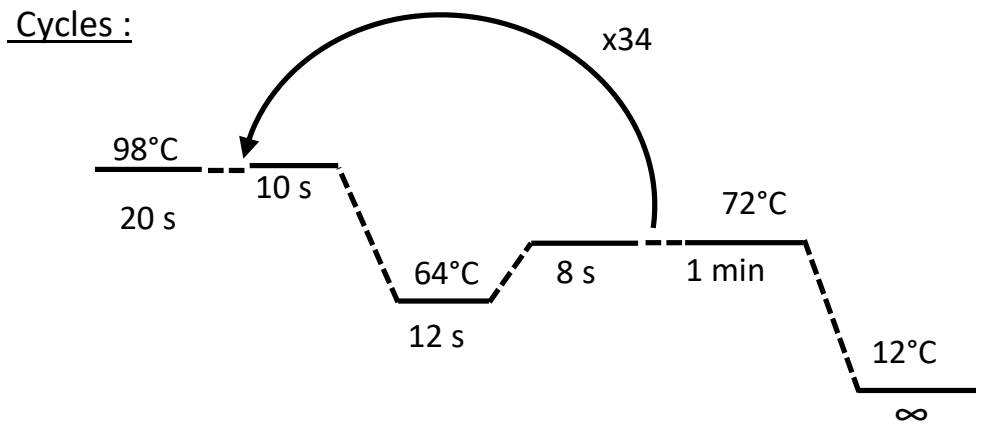

After amplification, the PCRs were treated with Dpnl for $1 \mathrm{~h}$ at $37^{\circ} \mathrm{C}$, diluted with $200 \mu \mathrm{L}$ of deionised water and purified by spin column purification (PCR purification kit from Macherey Nagel).

\section{Golden Gate assembly reactions}

\begin{tabular}{|c|c|c|}
\hline & Stock solution concentration & Volume/tube \\
\hline Purified digested PCR & typically $80 \mathrm{ng} / \mu \mathrm{L}$ & $2 \times 1 \mu \mathrm{L}$ or $3 \times 1 \mu \mathrm{L}$ \\
\hline Plasmid pCg69 or pg10 & $10 \mathrm{ng} / \mu \mathrm{L}$ & $1 \mu \mathrm{L}$ \\
\hline Cutsmart buffer (NEB) & $10 x$ & $1 \mu \mathrm{L}$ \\
\hline ATP & $10 \mathrm{mM}$ & $1 \mu \mathrm{L}$ \\
\hline Sapl & $10 \mathrm{kU} / \mathrm{mL}$ & $0.4 \mu \mathrm{L}$ \\
\hline T4 ligase & $400 \mathrm{kU} / \mathrm{mL}$ & $0.4 \mu \mathrm{L}$ \\
\hline \multirow[t]{3}{*}{ Nuclease free water } & - & $3.2 \mu \mathrm{L}(+1 \mu \mathrm{L})$ \\
\hline & & $10 \mu \mathrm{L}$ \\
\hline & $65^{\circ} \mathrm{C}$ & \\
\hline
\end{tabular}

The reaction can be inserted into competent $E$. coli without purification. Typically, this procedure yields about 100 to 300 clones for $5 \mu \mathrm{L}$ of reaction in $50 \mu \mathrm{L}$ of XL1 blue "home-made" competent cells. Correct assembly can be verified by digestion with Kpnl and Avrll which yields a band at 1427 bp for a successful 2-guide assembly and a band at 1962 bp for a 3-guide assembly (Vectors bands are at $4730 \mathrm{bp}$ and $8918 \mathrm{bp}$ for pg10 and pCg69 respectively). Sequencing can be done with the primers GG_For_S and Seq_guide. (See table1). 


\section{PCR on POX loci and SPS loci}

Y. lipolytica colonies were grown in YPD in microtiter plates sealed with a breathable membrane (Corning) at $28^{\circ} \mathrm{C}$, shaking at $600 \mathrm{rpm}$ for $24 \mathrm{~h}$. Samples (typically $50 \mu \mathrm{L}$ ) were centrifuged and the cells were washed with physiological water, centrifuged again and the resulting pellets were suspended in alkaline solution ( $\mathrm{NaOH} 25 \mathrm{mM}, 50 \mu \mathrm{L}$ ), prior to being heated at $95^{\circ} \mathrm{C}$ for $10 \mathrm{~min}$. Supernatants could be stored at $-20^{\circ} \mathrm{C}$ or used directly for matrix in the PCR reactions. PCR amplifications were done on the colony lysis solutions, with the following conditions using CloneAmp HiFi PCR Premix. See table S1 for primer sequences.

Lysis supernatant $\quad 2 \mu \mathrm{L}$

primers

7.5 pmol each

CloneAmp HiFi PCR Premix (2X) $12.5 \mu \mathrm{L}$

Total reaction volume

$25 \mu \mathrm{L}$

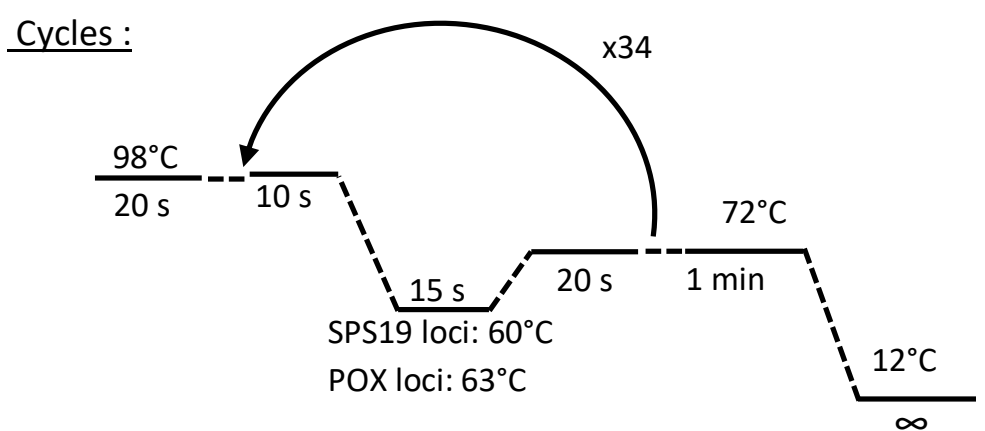

\section{PCR for the amplification of LEU2 and URA3 markers}

DNA was amplified from a JMP62-LEU2-ex-pTEF-dsRedStar or a JMP62-URA3-ex-pTEF-dsRedStar, primer sequences are given in SI table S1

Plasmids

primers

$20 \mathrm{ng}$

CloneAmp HiFi PCR Premix (2X)

Total reaction volume

$7.5 \mathrm{pmol}$ each

$12.5 \mu \mathrm{L}$

$25 \mu \mathrm{L}$

PCR cycler parameters:

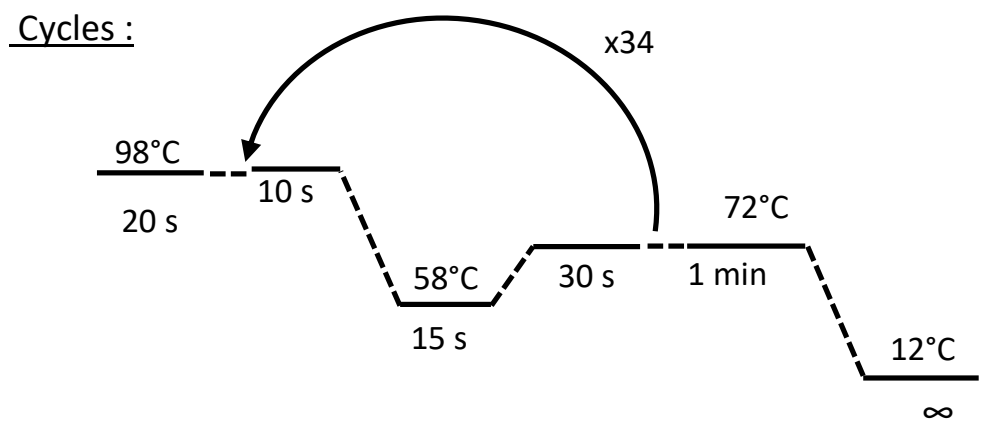

PCRs were column purified prior to transformation in $Y$. lipolytica. 


\section{Figure S1 Plasmids used in this study}

Plasmid maps were obtained with Snapgene.

\section{Generic plasmids for the Insertion of a single sgRNA}

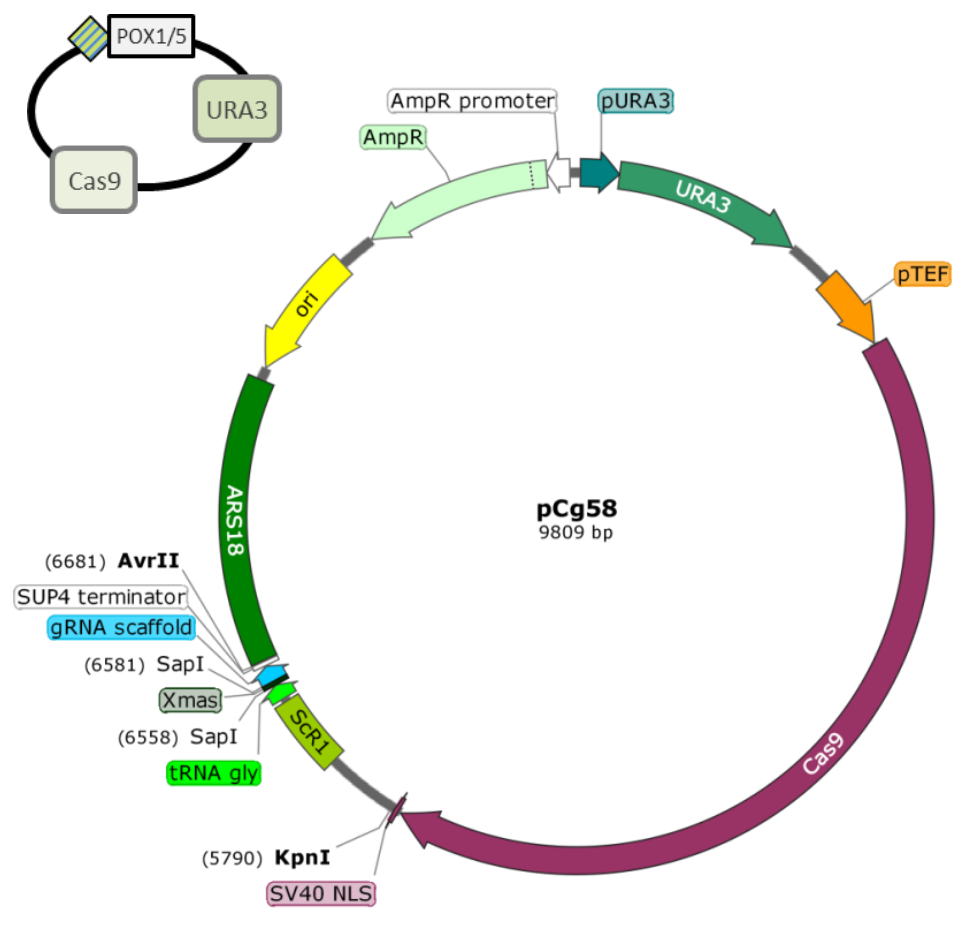

Plasmids directly derived from the insertion of target guide in place of the "Xmas" insert containing the two Sap/ sites:

$$
\begin{aligned}
& \text { - pCg58-POX1/5 } \\
& \text { - pCg58-POX4/6 } \\
& \text { - pCg58-SPS1a } \\
& \text { - pCg58-SPS2a } \\
& \text { - pCg58-SPS3a } \\
& \text { - pCg58-SPSm }
\end{aligned}
$$
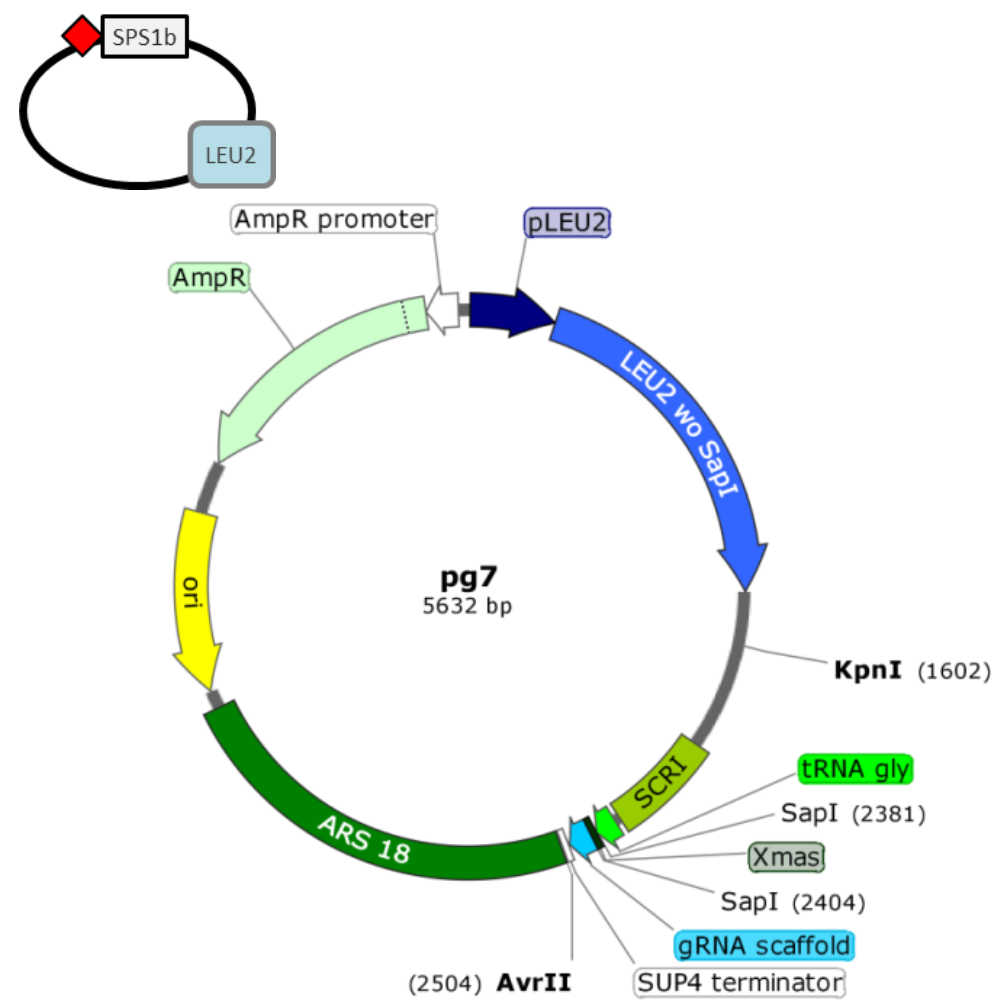

Plasmids directly derived from the insertion of target guide in place of "Xmas":

$$
\begin{aligned}
& \text { - pg7-SPS1b } \\
& \text { - pg7-SPS2b } \\
& \text { - pg7-SPS3b }
\end{aligned}
$$




\section{Generic plasmids for the Insertion of multiple sgRNA}

Insertion of amplicon takes place between the two Sapl sites.

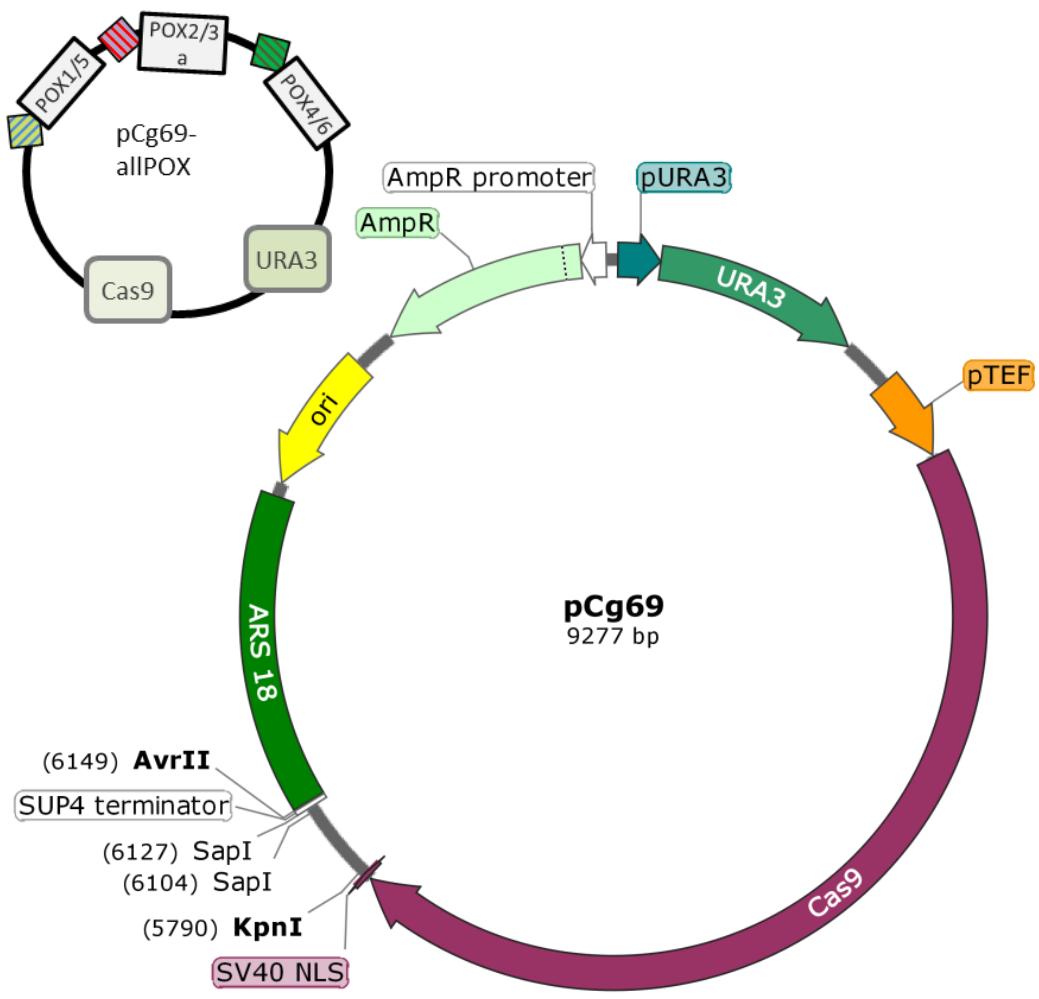

2-guide plasmids:

- pCg69-POX2/3:

$\mathrm{POX} 23 \mathrm{a}+\mathrm{POX} 23 \mathrm{~b}$

- pCg69-POX1456:

$\mathrm{POX} 1 / 5+\mathrm{POX} 4 / 6$

- pCg69-SPS191:

SPS1a + SPS1b

- pCg69-SPS192

SPS2a + SPS2b

- pCg69-SPS191b-SPS192b

SPS1b + SPS2b

3-guide plasmids:

- pCg69-allPOX:

POX1/5 + POX23a + POX4/6

- pCg69-SPS191/192

SPS1b+SPS2b+SPSm

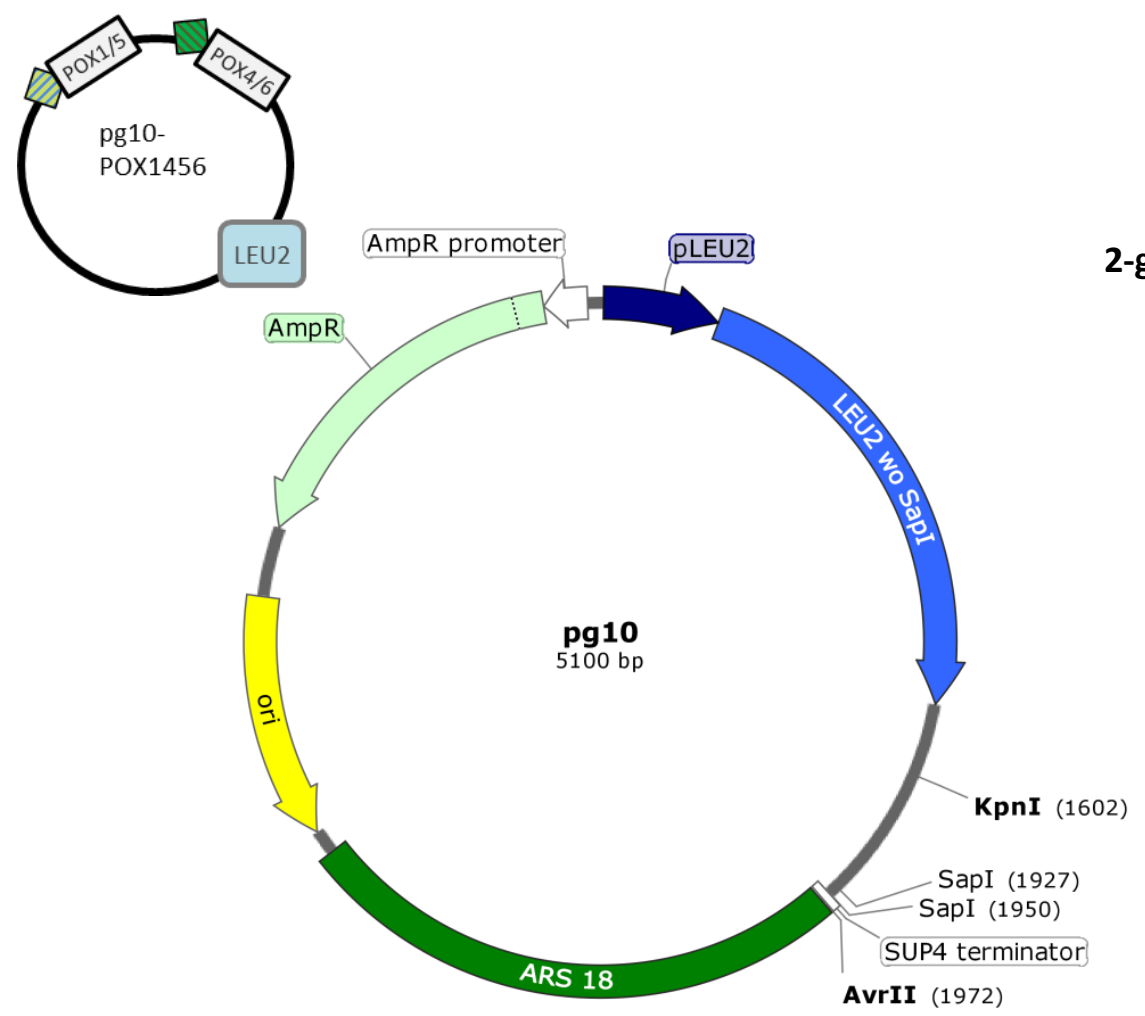

2-guide plasmids:

- pg10-POX1456

POX1/5+POX4/6

- pg10-SPS193

SPS3a+SPS3b 

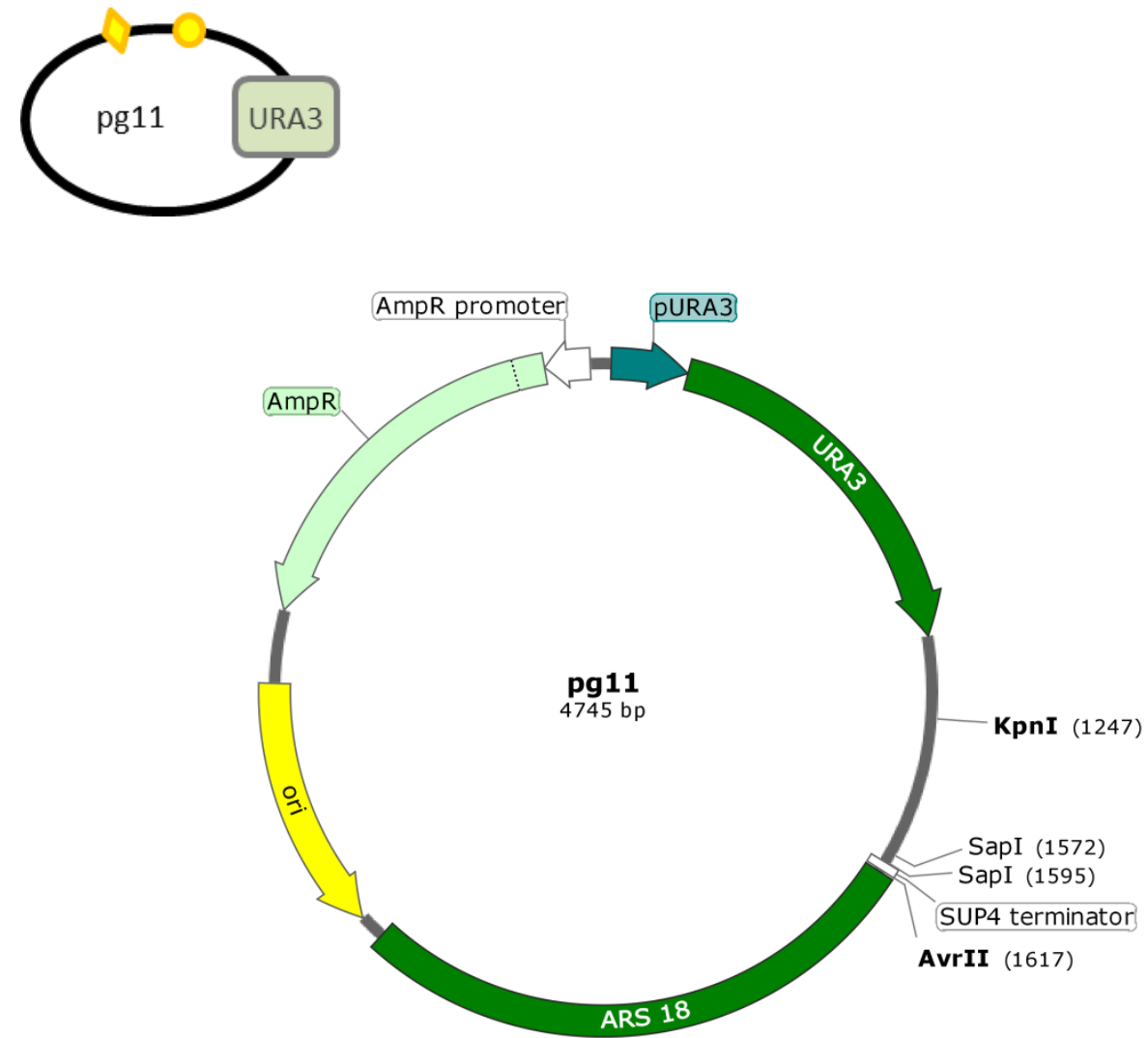

Used as a control plasmid for URA3 transformation marker 


\section{Figure S2 Primer design for multiplex assembly fragments}

\section{Amplicon 1:}

GG_For_S : $\quad$ attGCTCTTCTTGAGAGCCTTCAACCCAT

GG_Rev_GGT : attGCTCTTCCACCACCGACTCGGTGCCACT

\section{Amplicon 2:}

GG_For_GGT: attGCTCTTCAGGTTTTTTCCCCAGTTGCAAAAGTTGAC

GG_Rev_TTC: attGCTCTTCGGAAAAAACCACCGACTCGGTGCCACT

\section{Amplicon 3:}

GG_For_TTC: attGCTCTTCATTCCCCAGTTGCAAAAGTTGAC

GG_Rev_E: attGCTCTTCAAAGCACCACCGACTCGGTGCCACT

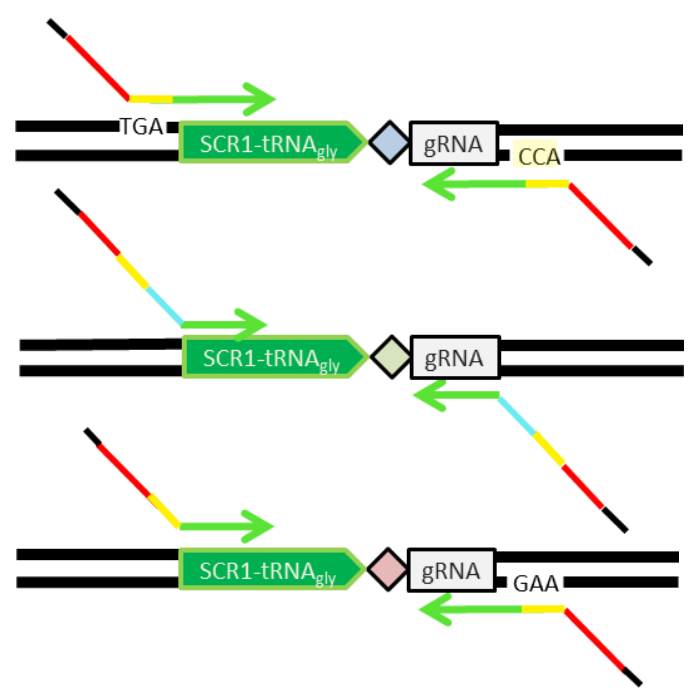

\section{Legend:}

Sapl site Bases involved in 5'overhang

Extra T added to introduce PollII terminator Hybridation to template

The combination shown above can be used for the construction of 3-guide plasmids.

For 2-guide plasmid, the following combination can be used:

\section{Amplicon 1:}

GG_For_S : attGCTCTTCTTGAGAGCCTTCAACCCAT

GG_Rev_GGT : attGCTCTTCCACCACCGACTCGGTGCCACT

\section{Amplicon 2:}

GG_For_GGT: attGCTCTTCAGGTTTTTTCCCCAGTTGCAAAAGTTGAC

GG_Rev_E: attGCTCTTCAAAGCACCACCGACTCGGTGCCACT

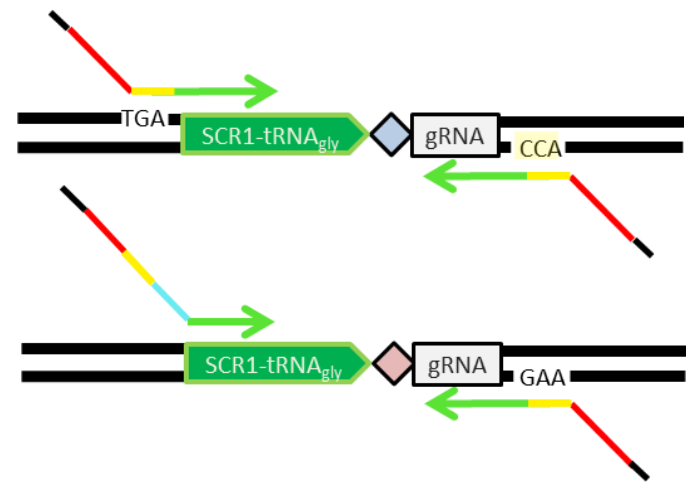




\section{Figure S3 DNA 90 nt oligomer design for HDR}

\section{POX1}

Ccacgtggggatgtgccaaatcattgatgagtgccga aggcctg AggTAA catggatactctggatataacggatttggccagggcta ccacgtggggatgtgccaaatcattgatgagtgccgacaggcctgtggaggtcatggatactctggatataacggatttggccagggctacg

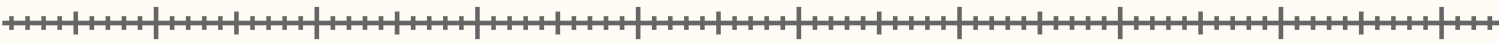
ggtgcacccctacacggtttagtaactactcacggctgtccggacacctccagtacctatgagacctatattgcctaaccggtcccgatgc

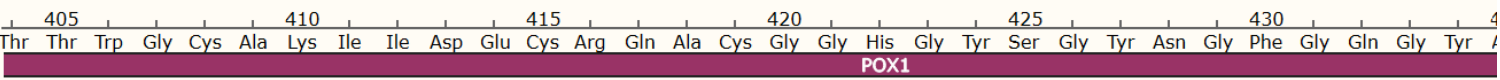

gPOX1/5

\section{POX4}

ggcttgtgctgacatcattgacaaggccogacaggcgtgatgaggcac TAa actctgcctacaacggctttggacaggccttccaggac :gggcttgtgctgacatcattgacaaggcccgacaggcgtgtggaggccacggatactctgcctacaacggctttggacaggccttccaggactgg $\mathbf{H} \mathbf{H}+\mathbf{H}+\mathbf{H}+\mathbf{H}+\mathbf{H}+\mathbf{H}+\mathbf{H}+\mathbf{H}+\mathbf{H}+\mathbf{H}+\mathbf{H}+\mathbf{H}+\mathbf{H}+\mathbf{H}+\mathbf{H}+\mathbf{H}+\mathbf{H}+\mathbf{H}+\mathbf{H}+\mathbf{H}+\mathbf{H}+\mathbf{H}+\mathbf{H}+\mathbf{H}+\mathbf{H}+\mathbf{H}+\mathbf{H}$ icc cgacacgactgtagtaactgttccgggctgtccgcacacctccggtgcctatgagacggatgttgccgaacctgtccggaaggtcctgacc

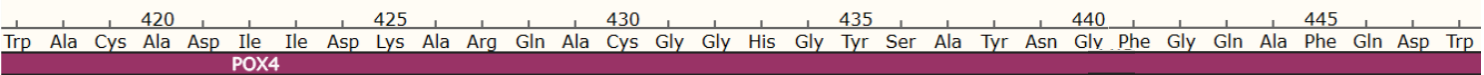

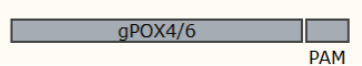

\section{POX5}

Ccacctgggctgccgccaagatcattgacgagtgccga aggcctg A A caccacctgggctgccgccaagatcattgacgagtgccgacaggcctgtggtggccatggctactccggctacaacggtt tcggtcaggcttac

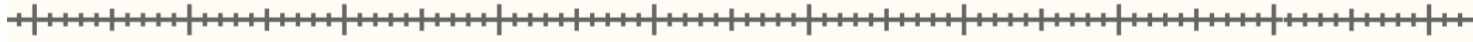
Igtggtggacccgacggcggttctagtaactgctcacggctgtccggacaccaccggtaccgatgaggccgatgttgccaagccagtccgaatg ie Thr Thr Trp Ala Ala Ala Lys Ile Ile Asp Glu Cys Arg Gln Ala Cys Gly Gly His Gly Tyr Ser Gly Tyr Asn Gly Phe Gly Gln Ala Tyr

\begin{tabular}{r|}
\hline gPOX $1 / 5$ \\
PAM
\end{tabular}

\section{P0X6}

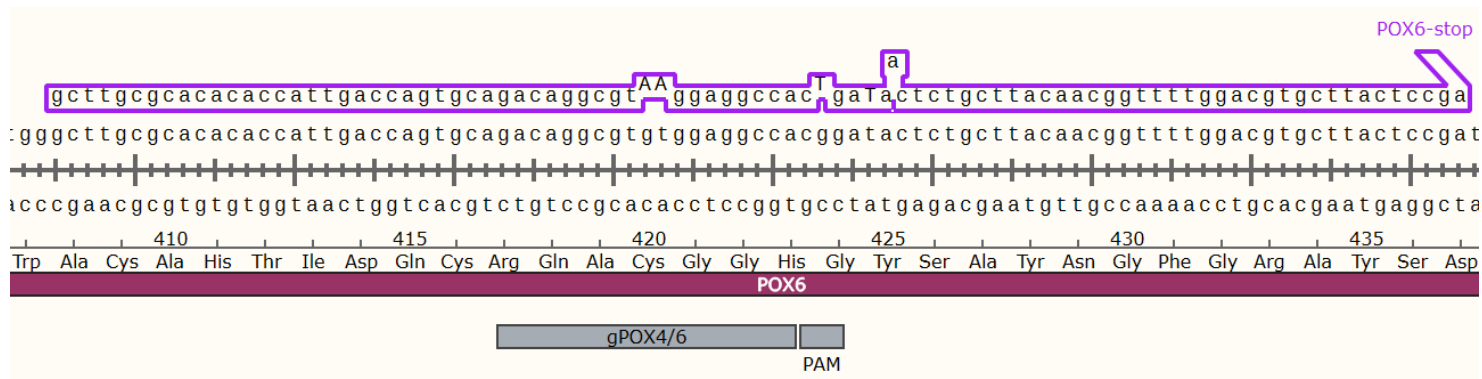

The nucleotides were conceived to remove the PAM sequence upon edition

The three bases in $3^{\prime}$ and $5^{\prime}$ of each oligonucleotide were protected by phosphorothioate linkages 
Figure S4 Comparison of transformation efficiencies between single and cotransformations

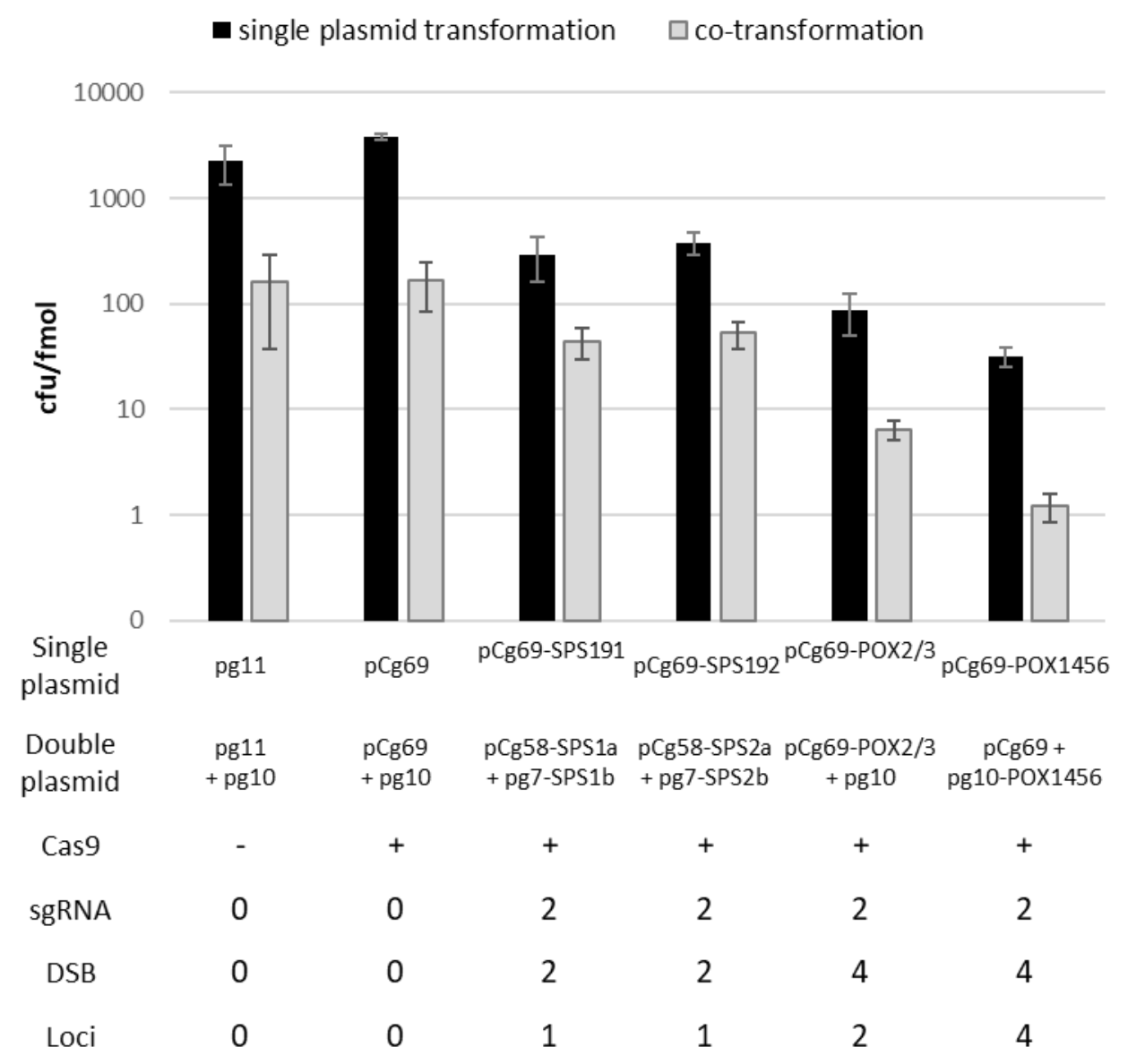

Effects of co-transformation on transformation efficiency. Identical combinations of sgRNA and Cas9 where introduced into Po1d competent cells by either single plasmid transformation (black bars) or co-transformation (grey bars) then survivors were counted. The results reflect the data from at least three different replicate experiments. 


\section{Figure S5 Toxicity of multiple sgRNA co-expression is associated to DSB}

formation

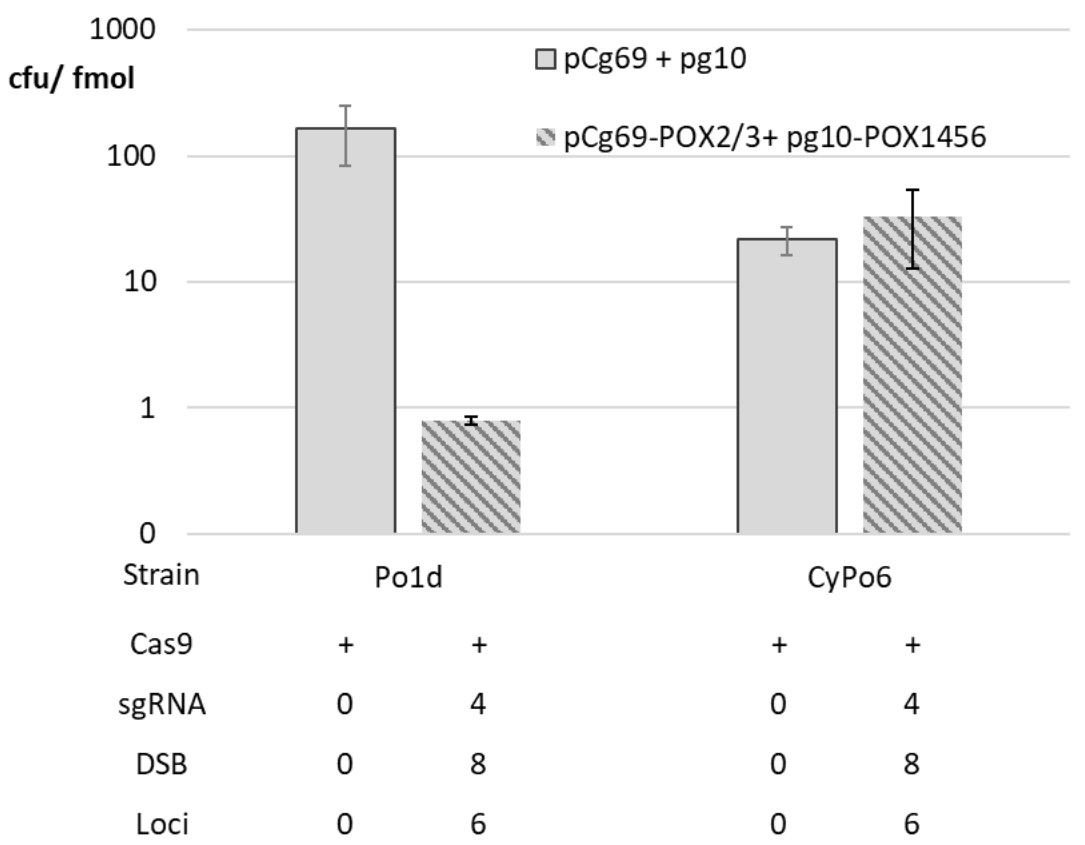

The same combination of plasmids (pCg69-POX-2/3 + pg10-POX1456), allowing the expression of Cas 9 and 4 sgRNAs, which targets a total of 6 genes and generates 8 simultaneous DSB, was transformed into two strains of competent cells. In Po1d, all targets are present, while in the CyPo6 strain, all targets have been previously removed by CRISPR/Cas9 editing. Transformation efficiency of Po1d, where DSB can be generated drops more than 100-fold in comparison to the control transformation without sgRNA ( $\mathrm{pCg} 69+\mathrm{pg} 10)$, whilst in CyPo6 where DSBs cannot be generated by the sgRNAs, transformation efficiency remains similar to the control. The results reflect the data from at least three different experiments. 


\section{Figure S6 Chromosomal translocations}

Example of translocations in CRISPR/Cas9 modified strain. When Colony PCR failed with the standard forward/reverse primer combination, the combinations were modified to look for translocations. Positive amplifications were sequenced. In the chromatograms were the junction were sequenced, the bases contained in the target sequence for the sgRNA are boxed. Alignments were obtained with Snapgene.

\section{Figure S6.1 Gels and chromatograms of POX translocations} WT:

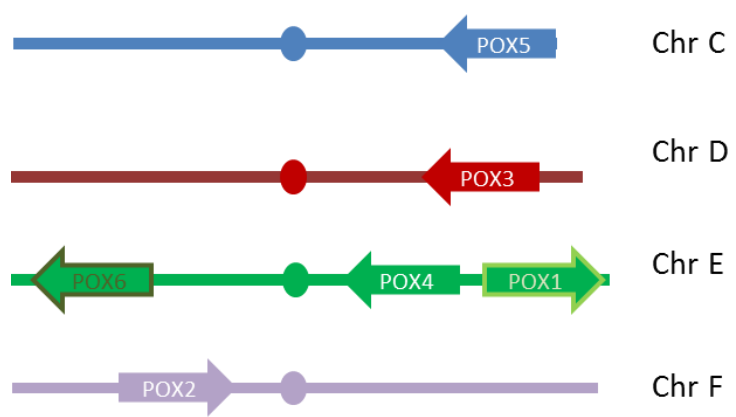

Figure S6.1.1 Translocations POX4/POX6

Gel PCRs Colony P46_10B, P46_10F, P46_10H, and P1456_5A
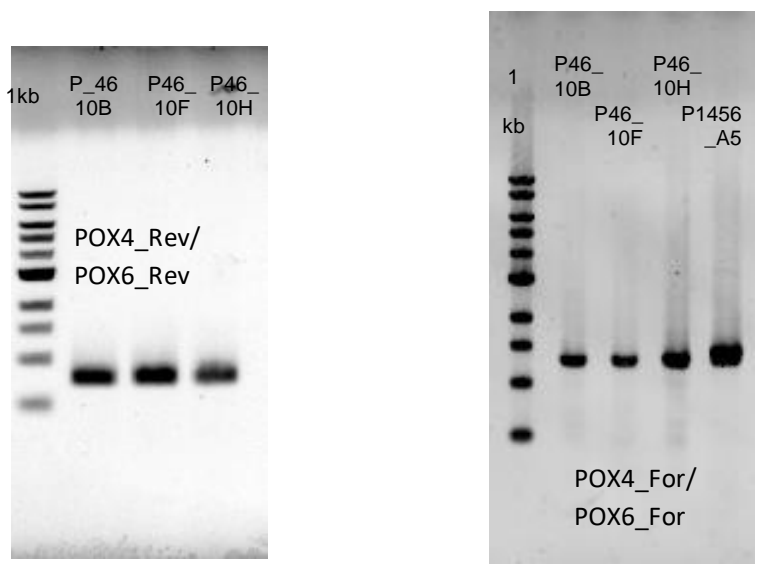

ChrE:

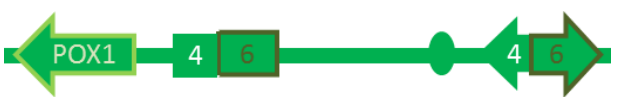

:CAGGTGCACTGGATCACCCAATCGGAGTAAGACGTCCAAAACCGTTGTAAGCAGAGTATCCGGGCACGGATACTCTGCCTACAACGGCTTTGGACAGGCCTTCCAGGACTGGGTTGTCCAGTGCAC'

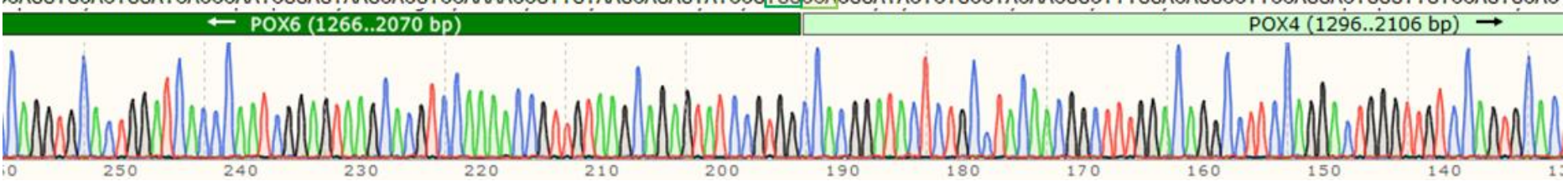

Chromatogram obtained with a PCR with POX4_Rev/POX6_Rev - Colony. P46_10B

AAGGCTGCCACTACATGGGCTTGTGCTGACATCATTGACAAGGCCCGACAGGCGTGTGGAGGCCTCCACACGCCTGTCTGCACTGGTCAATGGTGTGTGCGCAAGCCCAAGTTGTGGTAG POX4 (1.1295 bP) POX6 (1..1265 BP)

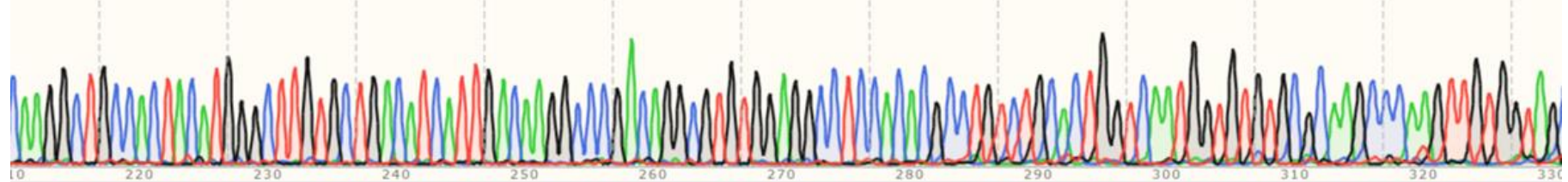

Chromatogram obtained with a PCR with POX4_For/POX6_For - Colony. P46_10B 
Figure S6.2.1 Translocations POX1/POX5

Gel PCRs Colony P15_5G:
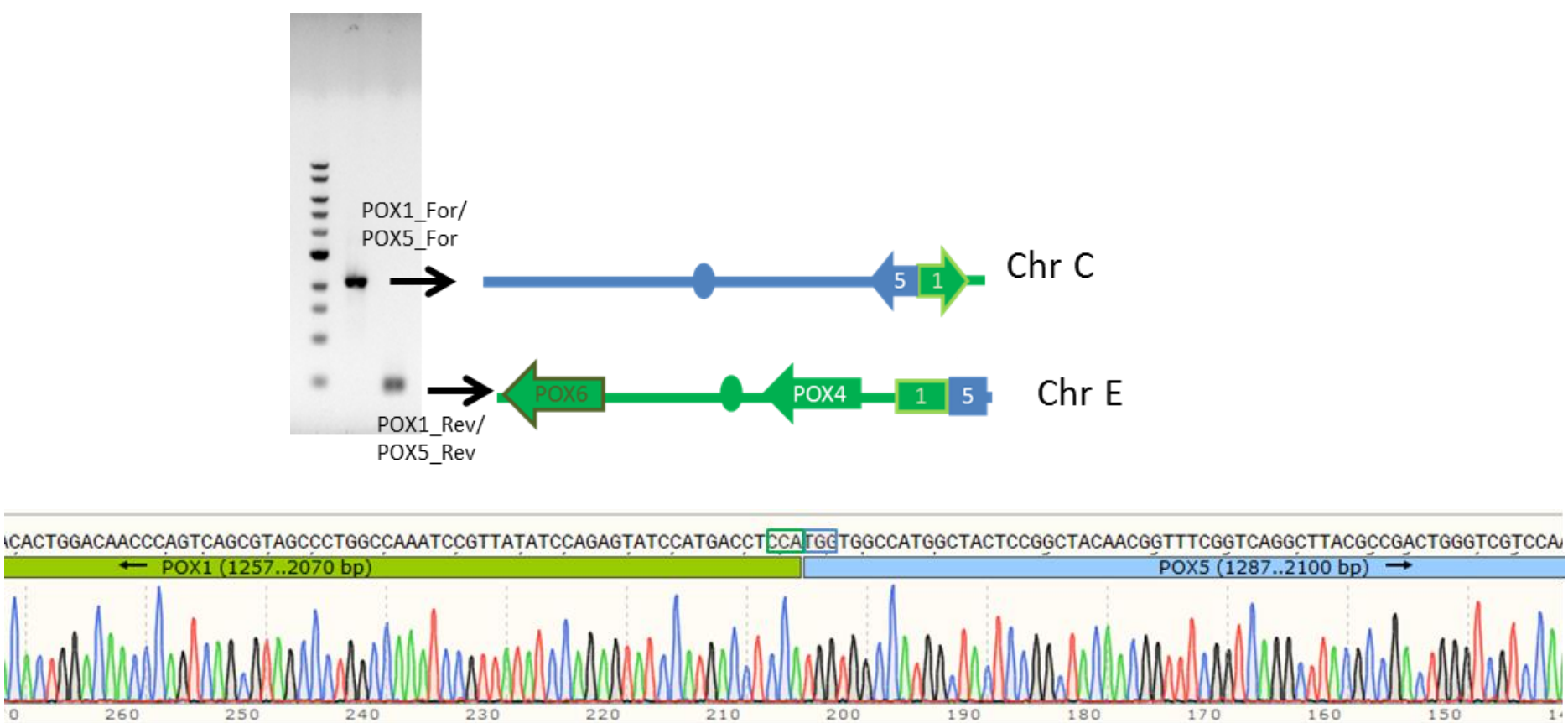

Chromatogram obtained with a PCR with POX1_Rev/POX5_Rev - Colony P15_5G

Figure S6.1.3 Translocations POX2/POX3

Chr D:

ACTGCCGAGCAGTTTGGCCACTGGATCTCCAAGGGAGCCGGAGACCTGCGAAAGTTCTACGGATGTTTCTCCATGACCAAGACTGGGTTGTCCAGTGCACCTGGGAGGGTGACAACAACGTCCTGTGTCTGTCCATGGGCCGAGGTCTS

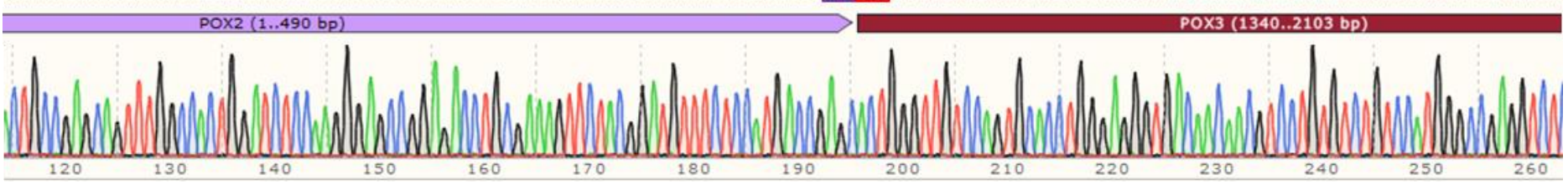

Chromatogram obtained with a PCR with POX2_For/POX3_Rev - Colony P23_2B

Chr F:

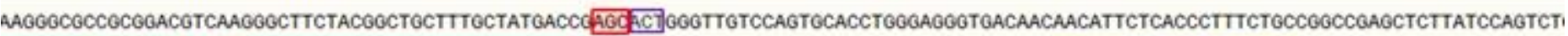
POX3(1..493 bP) POX2 (1337..2103 bp)

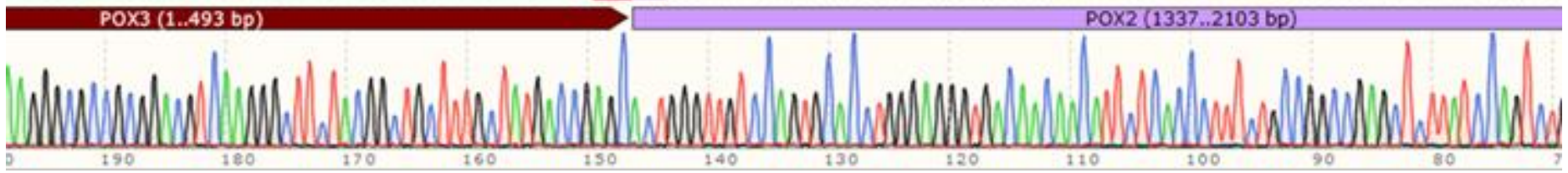

Chromatogram obtained with a PCR with POX3_For /POX2_rev-Colony P23_1D 


\section{Figure S6.1.4 Double reciprocal Translocations in P1456_A12 colony}

Colony P1456_A12:

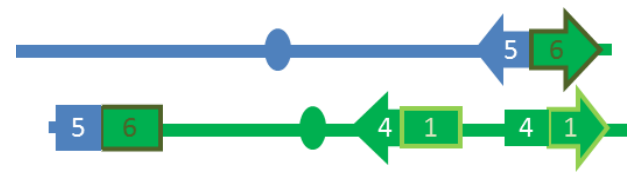

ACACTGGACAACCCAGTCAGCGTAGCCCTGGCCAAATCCGTTATATCCAGAGTATCCATGACCTCCATGGTGGCCATGGCTACTCCGGCTACAACGGTTTCGGTCAGGCTTACGCCGACTGGGTCGTCCA

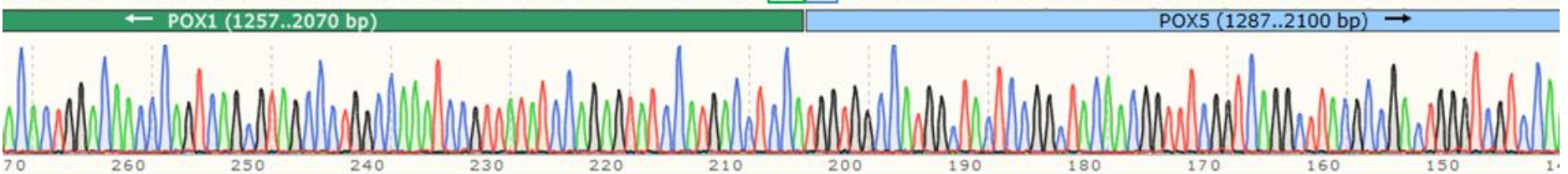

Chromatogram obtained with a PCR with POX5_Rev/POX6_Rev - Colony P1456_A12

TGGGCTGCCGCCAAGATCATTGACEAGTGCCGACAGGCCTGTGTTAACCAGCCTGTG ... ITCCATTGCATTECTCCACACGCCTGTOTGCACTGGTCAATGGTGTGTGCGCAAGCCCAAGTTGTG

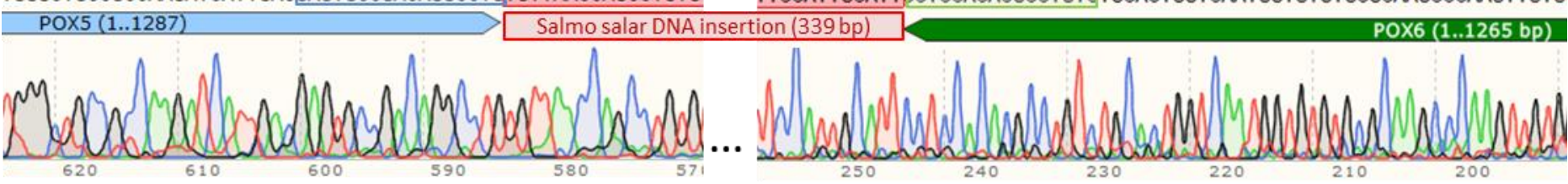

Chromatogram obtained with a PCR with POX5_For/POX6_For - Colony P1456_A12

GTTTGCTGCGTCTGCAGGAATGAAGGCATTTACCACGTGGGGATGTGCCAAAATCATTGATGAGTGCCGACAGGCCACGGATACTCTGCCTACAACGGCTTTGGACAGGCCTTCCAGGACTGGGTTTCCAGTGCACTTGGGAGGGTGACAAT

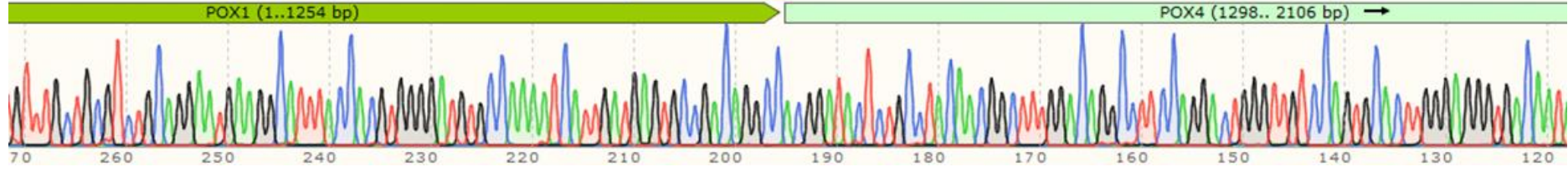

Chromatogram obtained with a PCR with POX1_For/POX4_Rev - Colony P1456_A12

CTGACATCATTGACAAGGCCCGACAGGCGTGTGGAGG 3 TGAGATGTTACCCCACACTTCCACCAAGGCACCTGCAAGTTTCCGGACACT GATACTCTGGATATAACGGATTTGGCCAGGGCTACGCTGACTGGG]

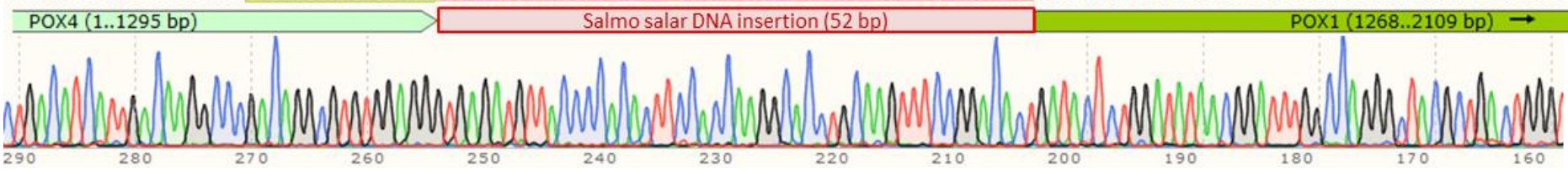

Chromatogram obtained with a PCR with POX4_For/POX1_Rev - Colony P1456_A12 
Figure S6.1.5 Translocations POX1/POX6

Chr E:

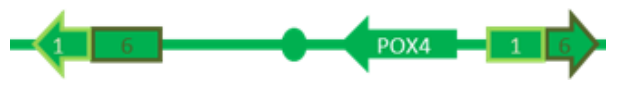

TGGTGTCAAGGCTACCACAACTTGGGCTTGCGCACACACCATTGACCAGTGCAEACAGGCGTGTGGAGGTGGAGTCATGGATACTCTGGATATAACGGATTTGGCCAGGGCTACGCTGACTGGGTTGTCCAGTGTACCTGGGAGGAC

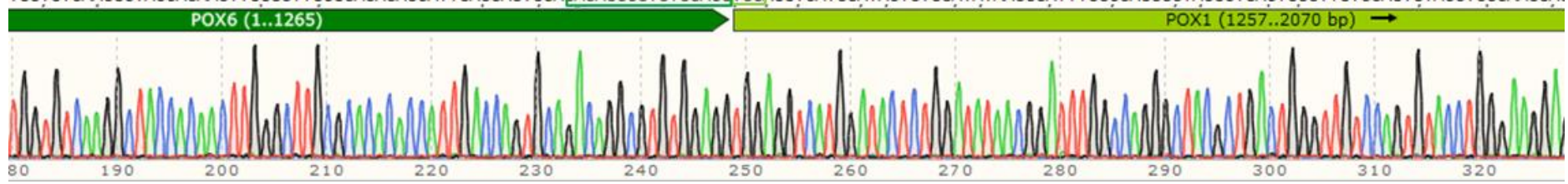

Chromatogram obtained with a PCR with POX1_Rev/POX6_ For - Colony P1456_E2

TGCGTCTGCAGGAATGAAGGCATTTACCACGTGGGGATGTGCCAAAATCATTGATGAGTGCCGACAGGCCTGCCAEGGATACTCTGCTTACAACGGTTTTGGACGTGCTTACTCCGATTGGGTGATCCAGTGCACCTGGGAGGGAGACA

Chromatogram obtained with a PCR with POX1_For/POX6_Rev - Colony P1456_E2 
Figure S6.2 Chromatograms of SPS translocations

WT

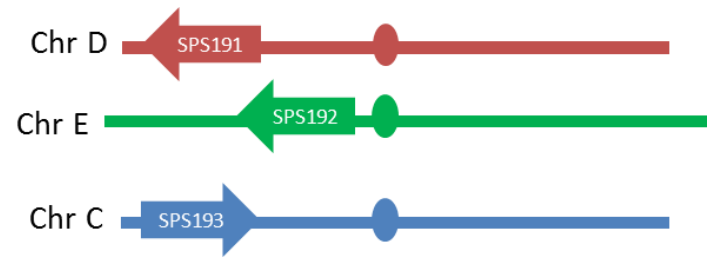

Figure S6.2.1 Translocation SPS192/SPS193(colony S23_4D)

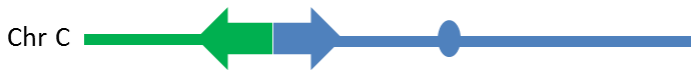

ICCGCATCAGAGAATAGAAACACGGTTCCGTTGGCAATGTCCTCTGTGGTTCCAAATCTCTGATGGGGCCAGACCGAGGACATTGCCAACGGTACCGTCTACGTCTTCTCCGAGGCCGCCTCTTACGTCTCCGGA

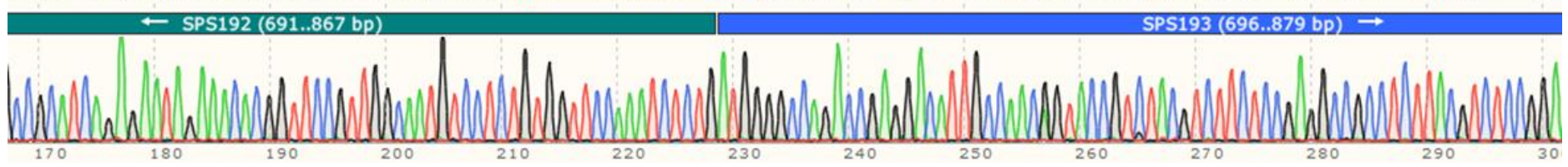

Chromatogram obtained with a PCR with SPS192_dwst/SPS193_Rev - Colony S23_4D
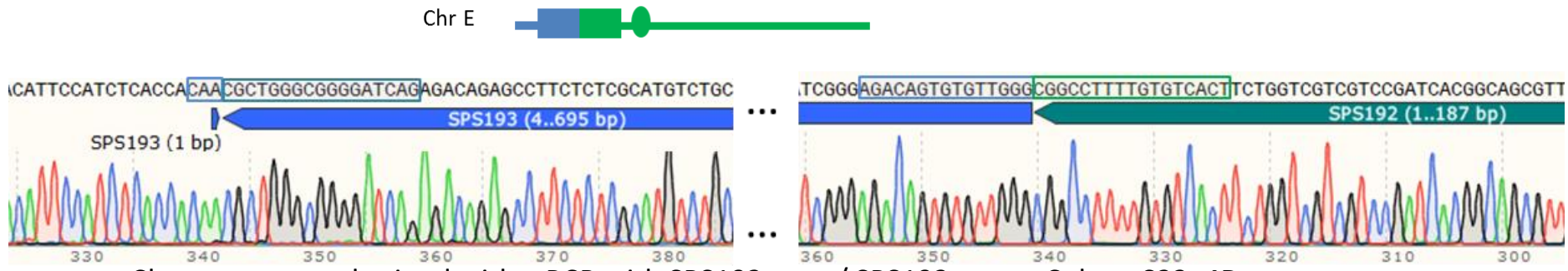

Chromatogram obtained with a PCR with SPS192_upst/SPS193_upst-Colony S23_4D

Figure S6.2.2 Translocations SPS191/192 (colony S123_12,S123_15)

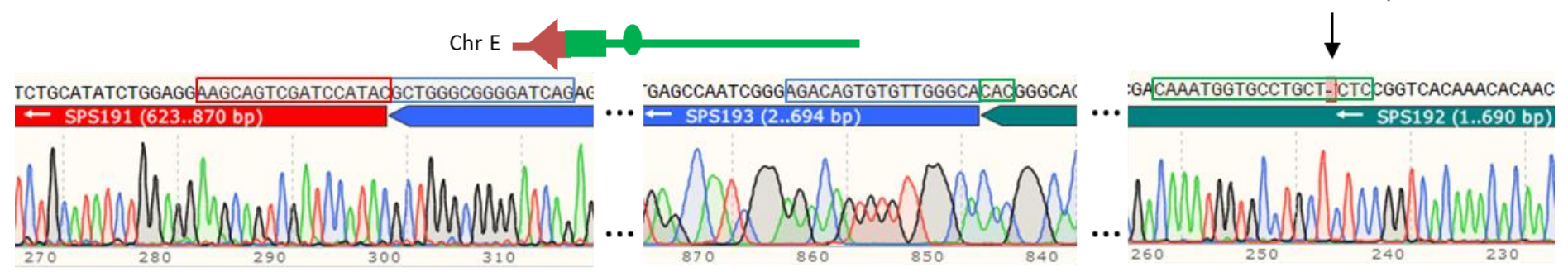

Chromatogram obtained with a PCR with SPS191_upst/ SPS192_dwst-Colony S123_15

Chr D

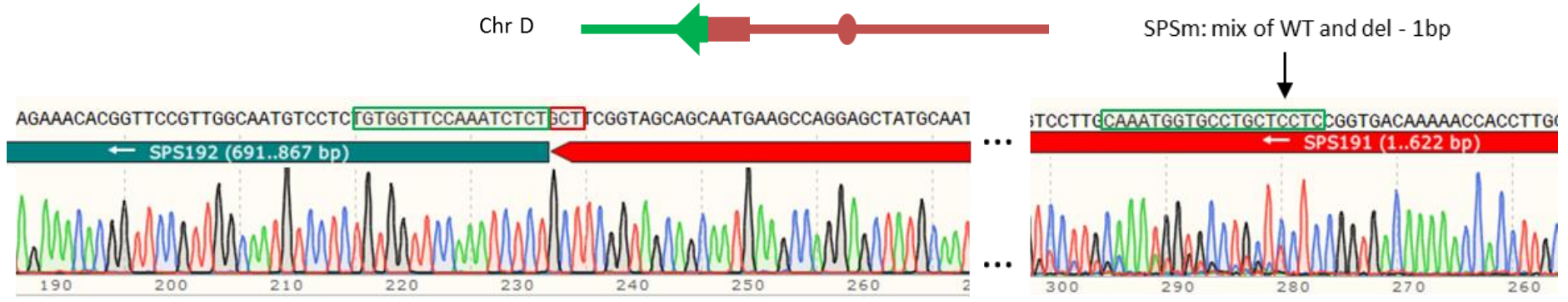

SPSm: mix of WT and del - 1bp

Chromatogram obtained with a PCR SPS191_dwst/ SPS192_upst-ColonyS123_15 
Figure S6.2.3 SPS191/193 (colony S13_7C, S13_7F, S123_17)

AGTATATGTCTTCTGCATATCTGGAGGAAGCAGTCGATCCATACCATGCTACCAGCAGATtTCATCGAGAGCTCGCCCTACAAGTCCA

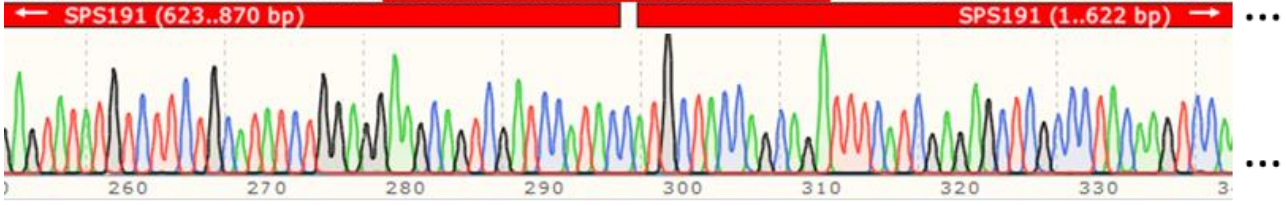

CTGCTACCG AAGATGGGGCAGACCGAGGACATTGCCAACG

Chromatogram obtained with a PCR SPS191_dwst/SPS193_Rev-Colony S13_7C

GACACTTTTAACGTCACCACCGCACATTCCATCTCACCACAATGTATGGAGAGTGCAAGTAGAGTTGAAGTTTCATGTGGGGTGGGGCATGCACGTGAAGTATTTGAGACAAGCCGTTGAGAGGGTACGGG

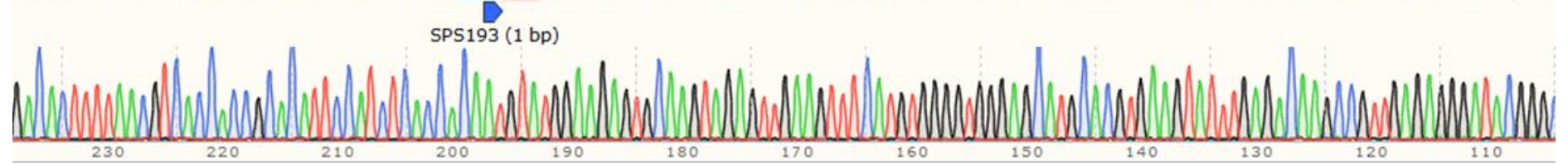

Chromatogram obtained with a PCR SPS191_upst/ SPS193_upst-Colony S13_7C 
Figure S6.2.4 Triple translocation (Colony S123_4)
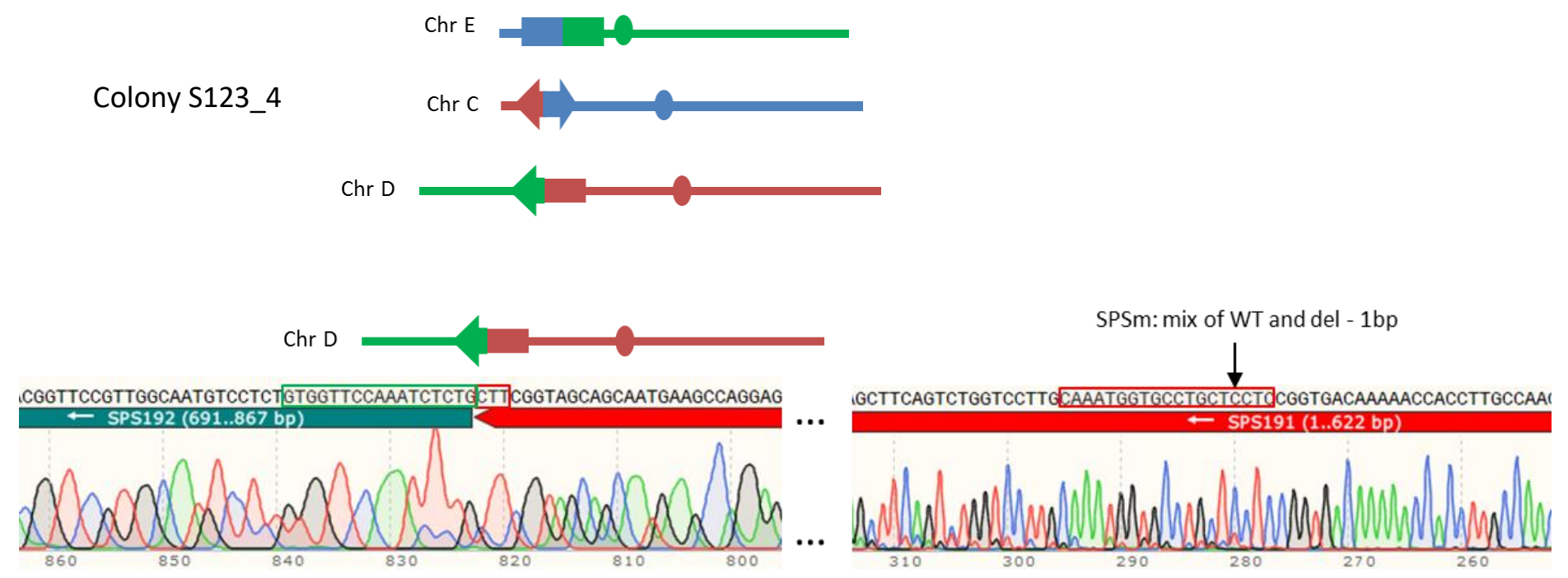

Chromatogram obtained with a PCR SPS191_upst/ SPS192_dwst-Colony S123_4

Chr E

mutation from WT sequence

SPS193 promoter region directly upstream of SPS3a_ 9 bp away from DSB
;TAACAGACACTTTTAACGTCACCACCGCACATTCCATTTCACTOCGGTCACAAACACAACCTTGCCATCAAACAGGCCGGGGTTGTAGACGCAGGTCTTGAGCCATTCGGGAGTAAGAGTGTTGGGCATGT

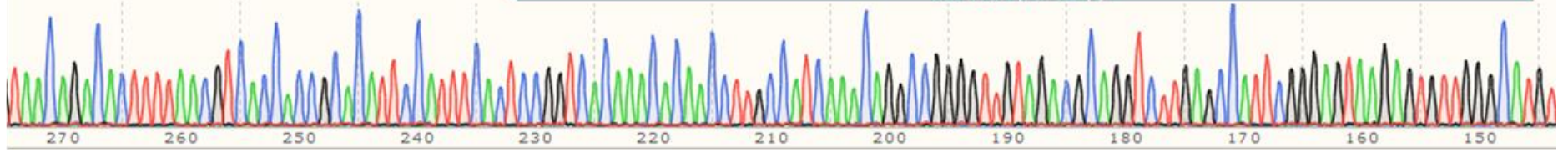

Chromatogram obtained with a PCR SPS193_upst/ SPS192_upst-Colony S123_4

Chr C

ICCGTTGCAGCGGCGTCAGACGAGTATATGTCTTCTGCATATCTGGAGG AAGCAGTCGATCCATAATGGGGCCAGACCGAGGACATTGCCAACGGTACCGTCTACGTCTTCTCCGAGGCCGCCTCTTACGTC

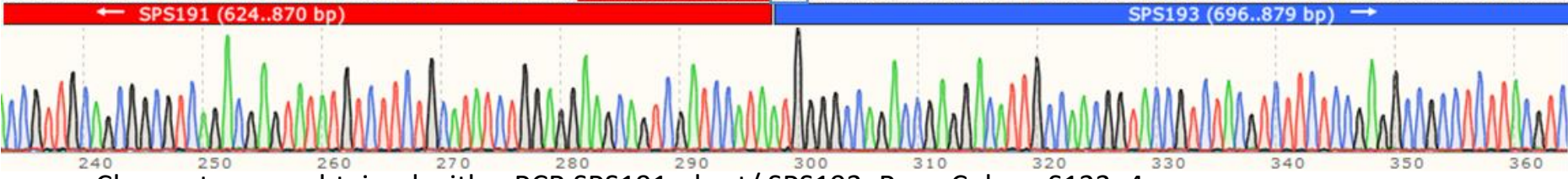

Chromatogram obtained with a PCR SPS191_dwst/ SPS193_Rev-Colony S123_4 


\section{Single DSB gene targeting}

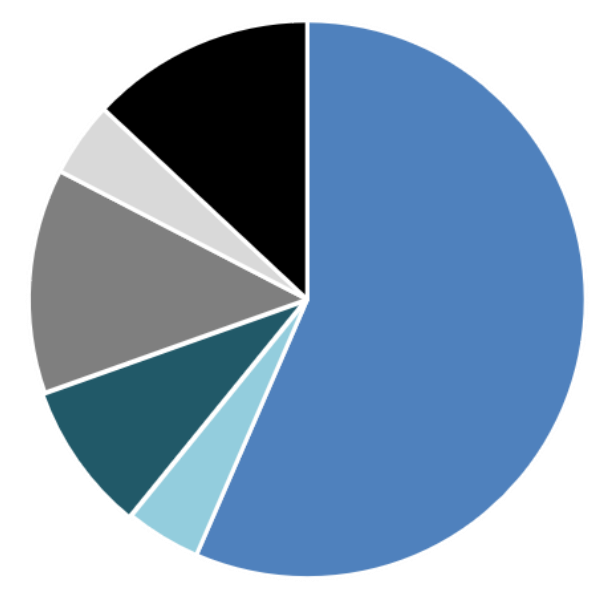

Clean junction

Undeleted DNA ends and small insertions

Undeleted DNA ends and large insertions

\section{Double DSB gene targeting}

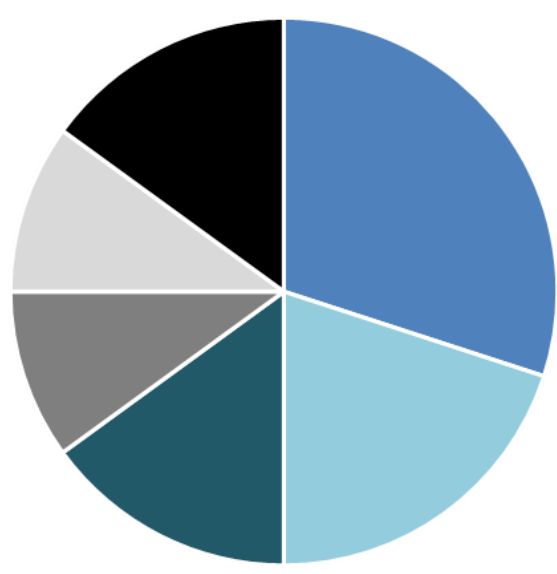

Deleted DNA ends and no DNA insertion

Deleted DNA ends and small insertions

Deleted DNA ends and large insertions

Sequence analysis of translocations junctions. The junctions that were successfully unambiguously sequenced were classified according to the deletion and insertion patterns at the DSB (SI Fig S6.1 and S6.2). The DNA ends were called "undeleted" when no base was missing from the CRISPR cut site (i.e. when $3 \mathrm{bp}$ of the guide sequence were left on the side of the PAM and 16 or $17 \mathrm{bp}$ on the opposite side of the PAM, depending on whether the guide was 19 or 20 bp-long). The term "clean junction" refers to genetic translocation events where both sides of the junction present unaltered DNA ends and no insertion in between (SI Fig S6.1.1 or S6.2.1, for instance). Small insertions of less than 3 bases (SI Fig S6.2.3) were differentiated from large insertions ranging from 40 bp to $700 \mathrm{bp}$ (SI Fig S6.1.4, Fig 56.2.2), are they are likely to have arisen through a different mechanism. A) The graphic on the left shows the data aggregation for POX1, POX4, POX5, and POX6 targeting experiments (single targeting). A total of 23 junction sequences were used (SI table S5.1 for detail). B) The graphic on the right is the aggregation of results from POX2, POX3 and SPS genes targeting (excision strategies). A total of 20 junction sequences were used (SI table S5.2 for detail). Sequence alignments of the insertions showed that they resulted from the inclusion of DNA carrier sequences (SI Fig S6.1.4) or an excised fragment (SI Fig S6.2.2) in experiments were excision strategies were used. 


\section{Figure S7 PCR screening of colonies by excision}

Loci were amplified as described in the "Protocols" section with the appropriate primers (see SI table 1). Pictures of the ethidium bromide stained gels were obtained with Image Lab and are presented in inverted colours for clarity purposes.

Figure S7.1- POX2 and POX3 genes excisions

\section{Expected lengths of amplicons}

\begin{tabular}{ccc}
\hline Loci & WT/ flip/ NHEJ & Excision a/b \\
\hline POX2 & $1249 \mathrm{bp}$ & $403 \mathrm{bp}$ \\
POX3 & $1219 \mathrm{bp}$ & $373 \mathrm{bp}$
\end{tabular}

Gel of clones P23.

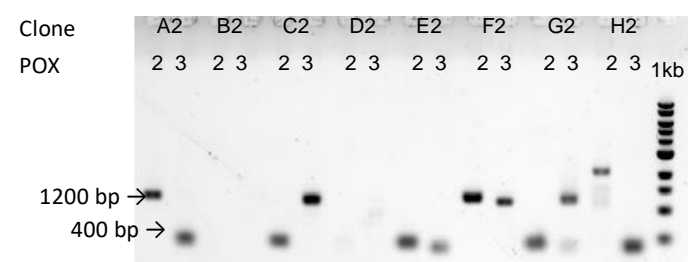

Absence of bands in colony P23_B2 and P23_D2 lanes occurred as a result of chromosomal translocations.

Figure S7.2- SPS19 gene excisions

Expected lengths of amplicons

\begin{tabular}{cccc}
\hline Loci & WT/ flip/NHEJ & Excision a/b & Excision $\mathbf{m} / \mathbf{b}$ \\
\hline SPS191 & $1178 \mathrm{bp}$ & $553 \mathrm{bp}$ & $635 \mathrm{bp}$ \\
SPS192 & $1118 \mathrm{bp}$ & $615 \mathrm{bp}$ & $516 \mathrm{bp}$ \\
SPS193 & $1254 \mathrm{bp}$ & $561 \mathrm{bp}$ & N. A.
\end{tabular}

\section{Mono-disruptant transformations screening}

pCg58-SPS1a + pg7-SPS1b

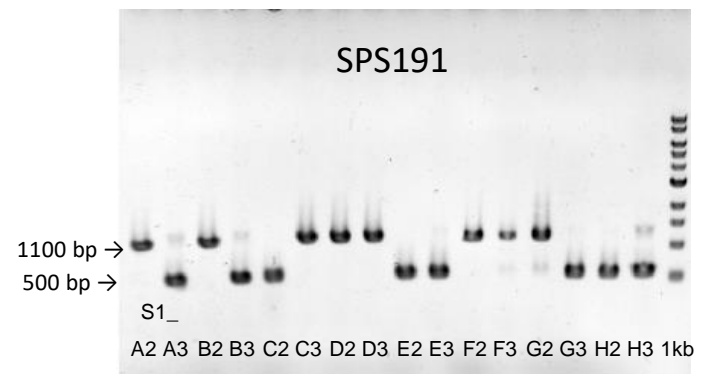

pCg58-SPS1a +pg7-SPS1b:

SPS192
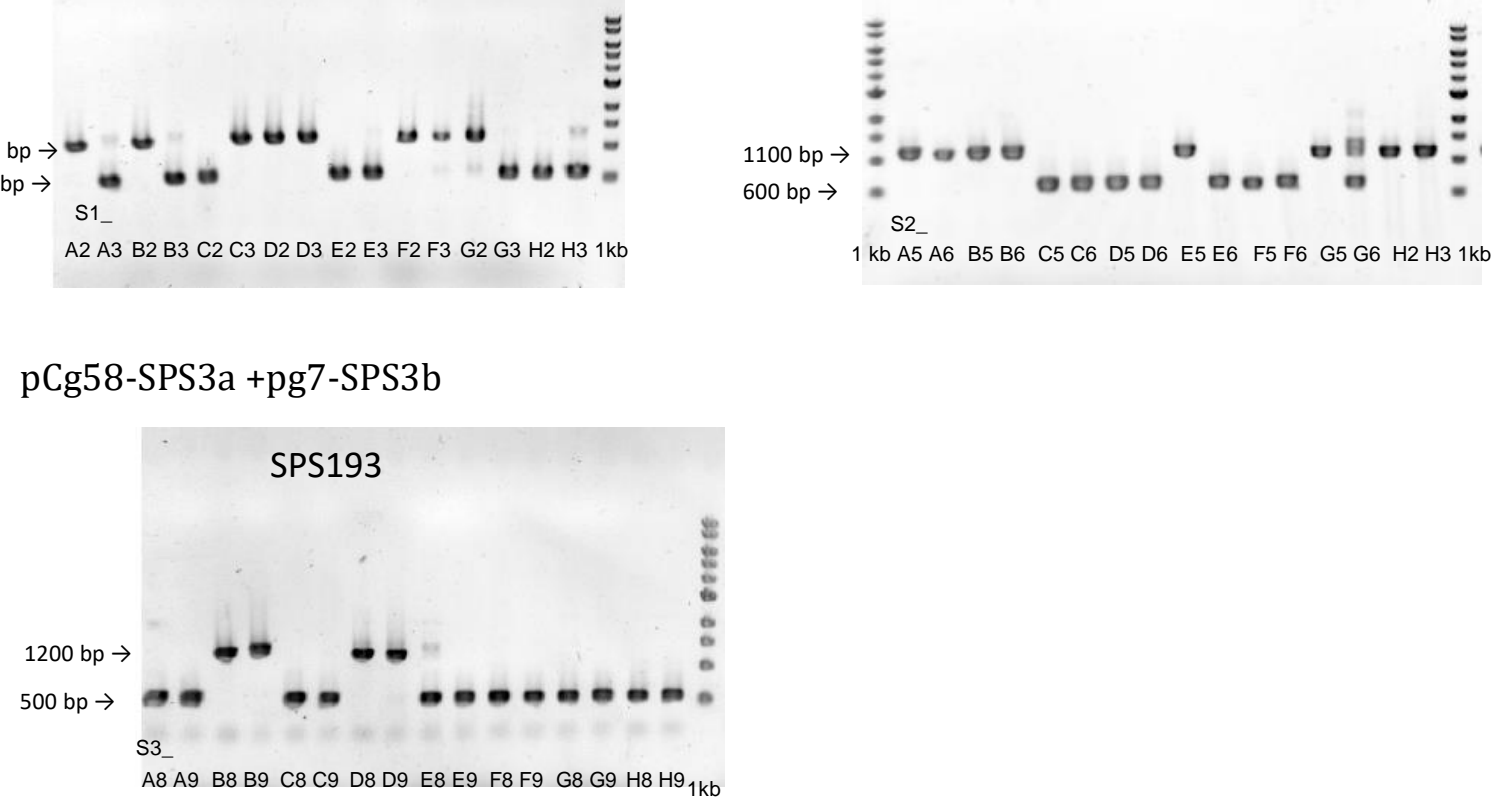


\section{Double-disruptant transformations screening}
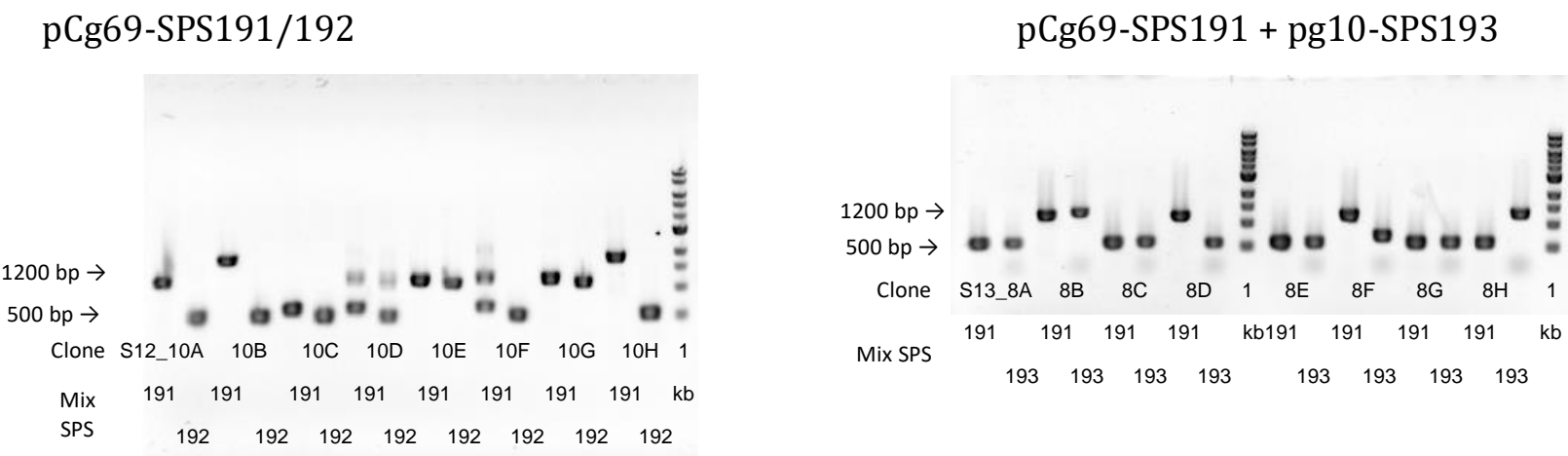

pCg69-SPS192 + pg10-SPS193

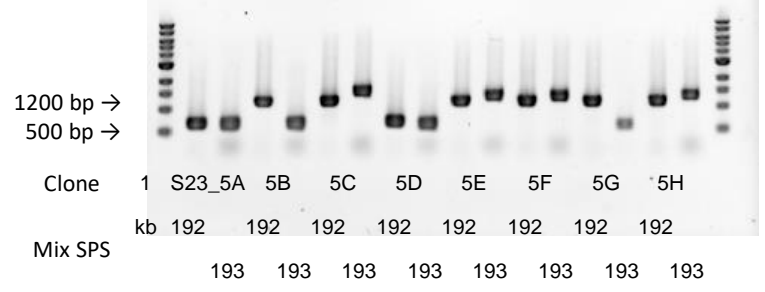

Triple-disruptant transformations screening

pCg69-SPS191/192 + pg10-SPS193

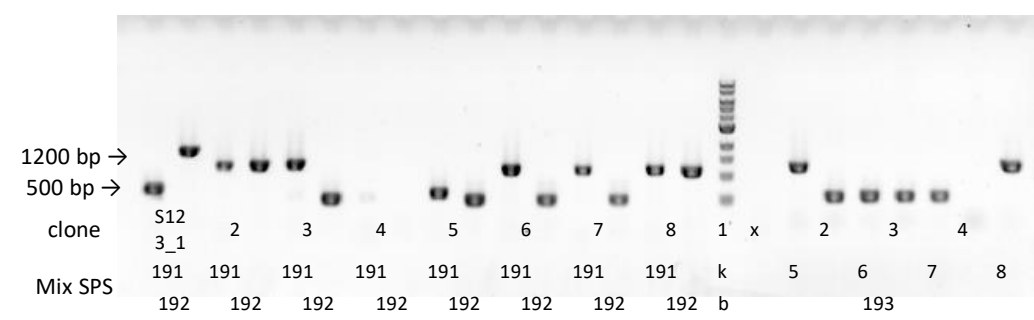

Absence of bands in colony S123_4 lanes occurred as a result of chromosomal translocations. 


\section{Figure S8-Differential growth of POX disruptants depending on fatty acid complementation}

A)

$\mathrm{OD}_{600 \mathrm{~nm}}$

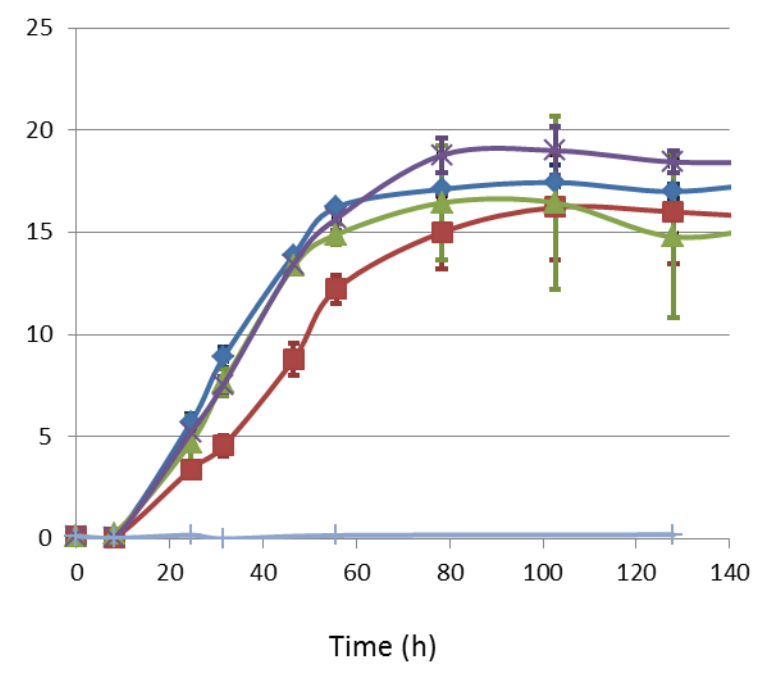

B)

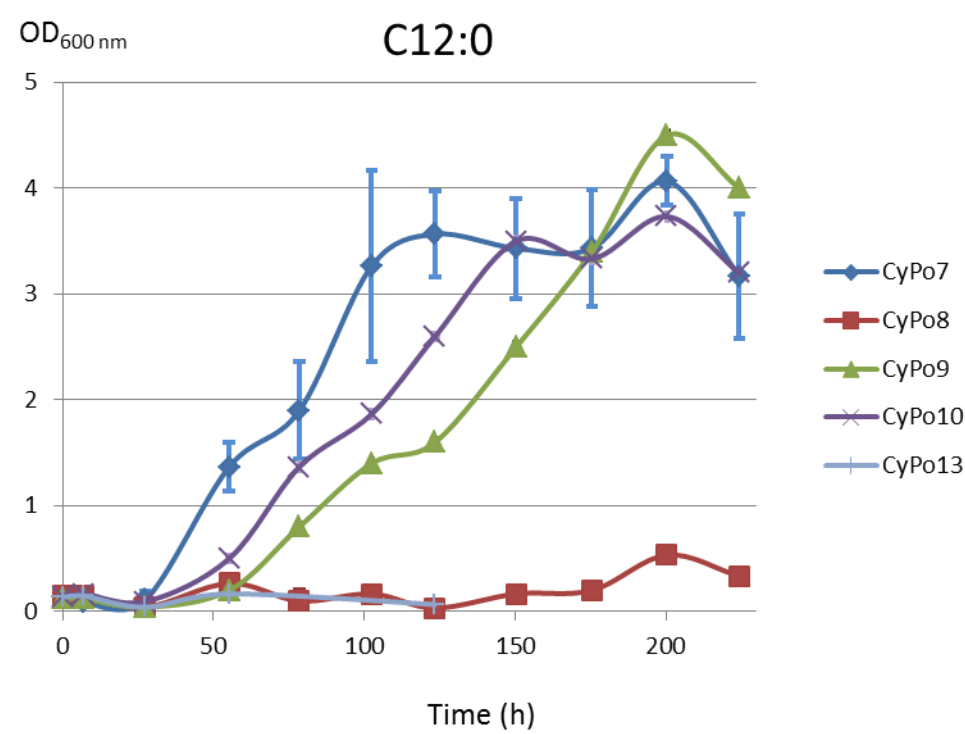

Individual colonies from CyPo7 (control), CyPo8 (pox2-, pox3-), CyPo9 (pox1-, pox5-), CyPo10 (pox4-, pox6-), and CyPo13 (pox1-, pox2-, pox3-, pox4-, pox5-, pox6-) transformation were picked and transferred to YPD medium. Three isolated transformants from each transformation were grown in culture tubes containing $5 \mathrm{~mL}$ of YPD, shaken at $28^{\circ} \mathrm{C}, 140 \mathrm{rpm}$, for $22 \mathrm{~h}$. The cultures were used to inoculate $20 \mathrm{~mL}$ of YNB medium supplemented with either C12:0 or C18:0 at $1 \mathrm{~g} / \mathrm{L}$ in $100 \mathrm{~mL}$ shake flask. The flasks were incubated in a shaking incubator (Infors) at $140 \mathrm{rpm}, 28^{\circ} \mathrm{C}$ and OD was monitored at $600 \mathrm{~nm}$ by diluting aliquots with a 1:3 mixture of water:IPA. Errors bars reflect the results obtained with three independent clones. 


\section{Figure S9 SPS19 genes family}

\section{Figure S9.1 SPS19 genes in Saccharomycotina}

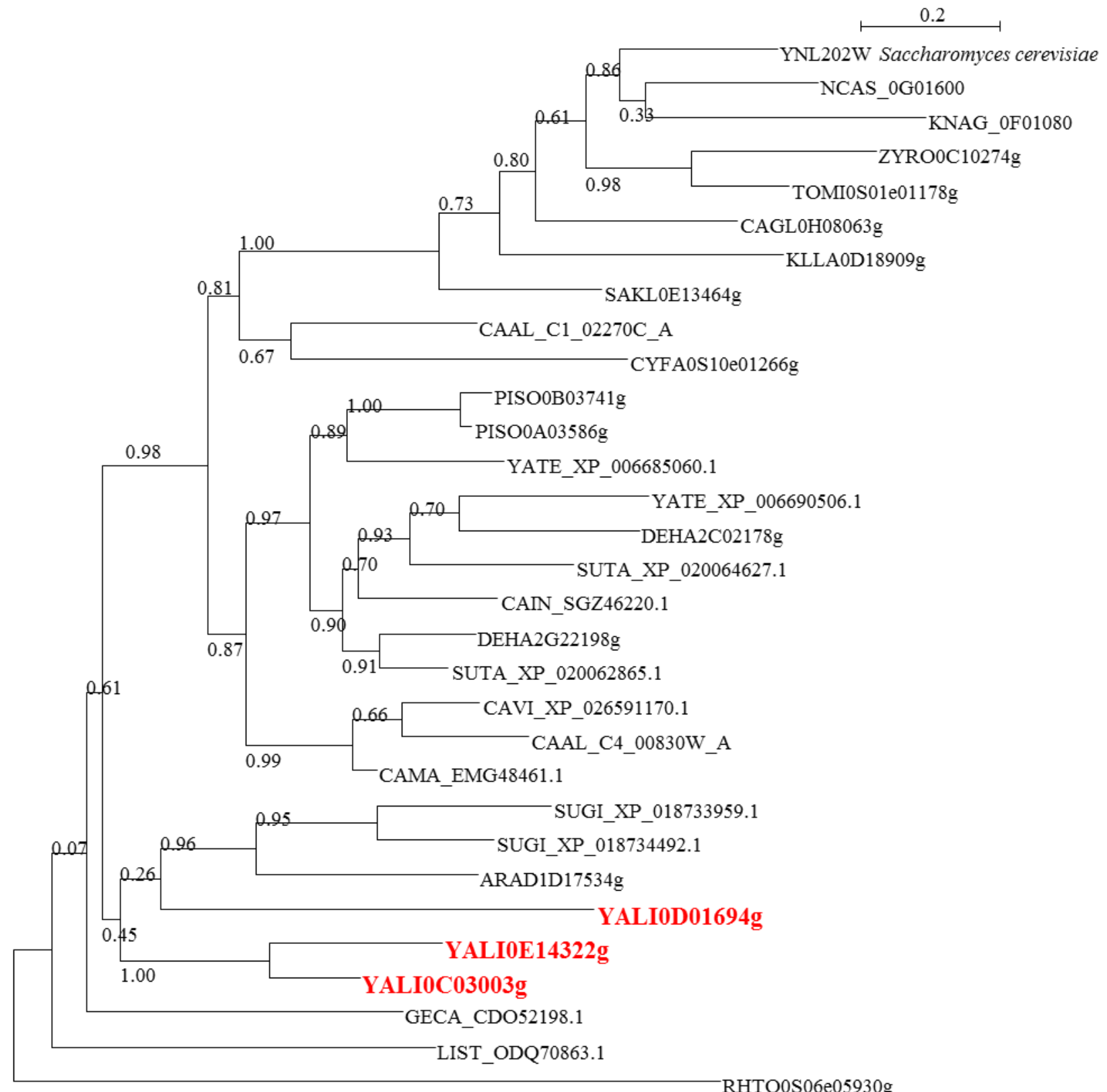

PhyML $\ln (\mathrm{L})=-9139.0309$ sites LG 4 rate classes

Phylogeny in Saccharomycotina shows that there is usually only one version of SPS19 gene per organism, and more rarely two, while $Y$. lipolytica has three. 


\section{Figure S9.2 Phylogeny of SPS19 genes in the Yarrowia clade}

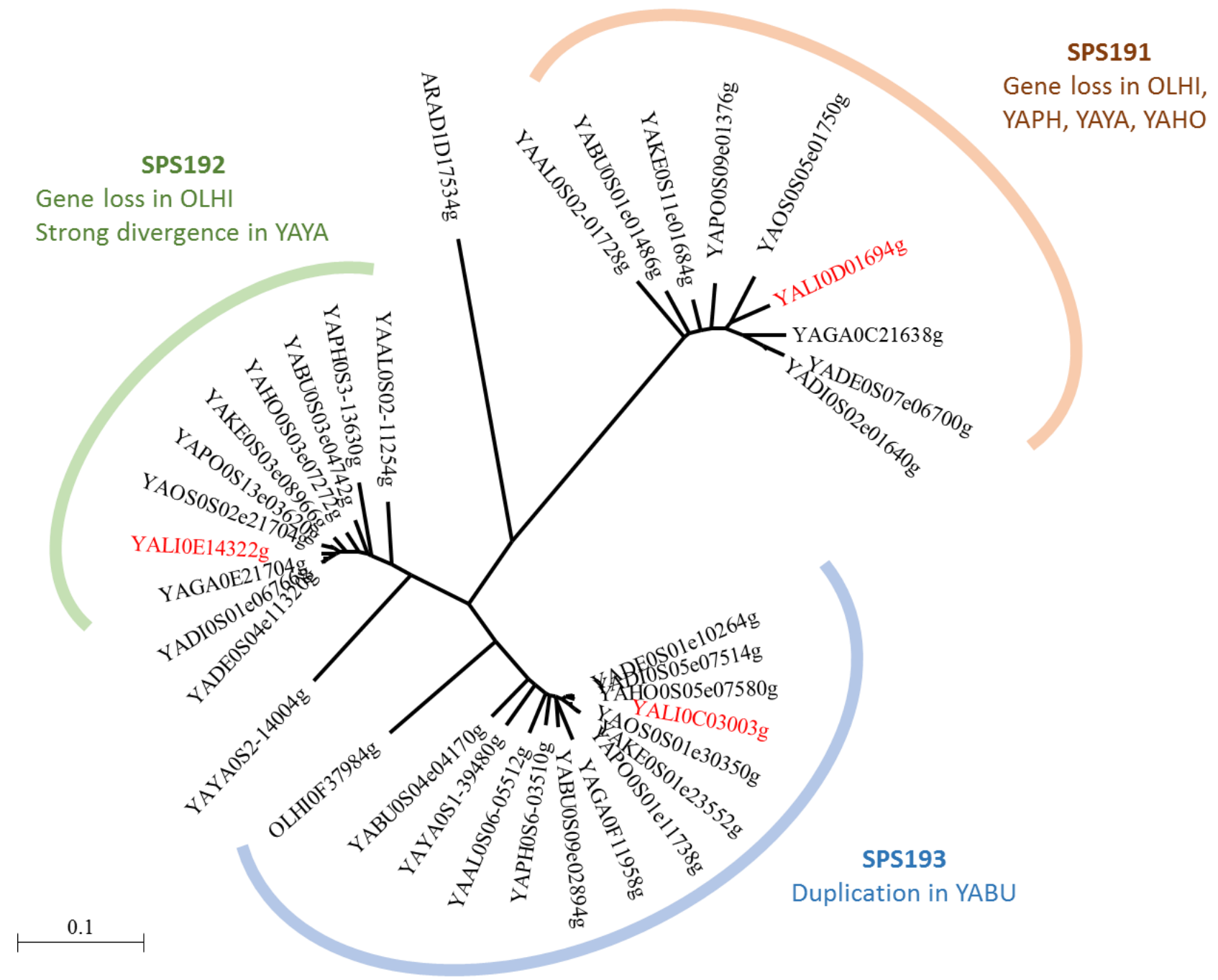

Phylogeny of SPS19 genes in the Yarrowia clade showing two ancestral duplications followed by species-specific losses and duplication 

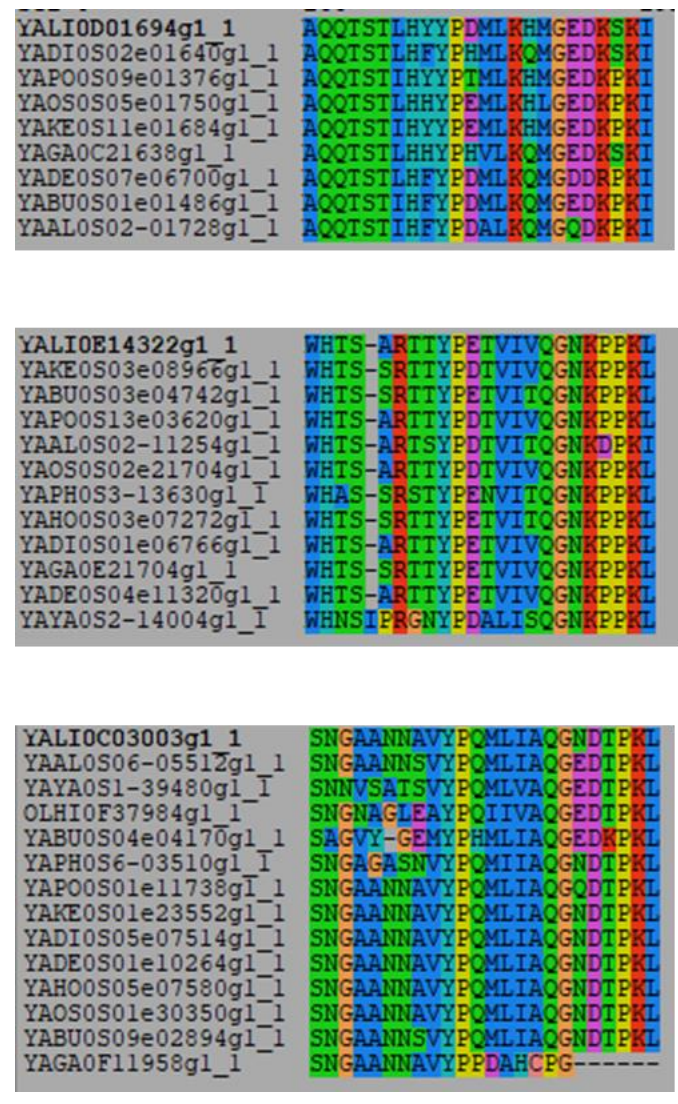

C-ter Motif

SPS191

$89-98 \%$ identity

$96-98 \%$ similarity

(S/P)KI
SPS192 PKL

$82-98 \%$ identity

91-99\% similarity

SPS193

$77-98 \%$ identity

$86-100 \%$ similarity
PKL

SPS19 genes show strong conservation in the C-term region with a PTS1 motif directing the protein to the peroxisome. 
Table S1 List of oligonucleotides used in this study

\begin{tabular}{|c|c|c|}
\hline \multicolumn{2}{|c|}{ Primers for PCR amplicons for Golden Gate assembly } & \multirow{7}{*}{$\begin{array}{l}\text { Template } \\
\text { pCg58 or pg7 with sgRNA in place }\end{array}$} \\
\hline GG_For_S & attGCTCTTCTTGAGAGCCTTCAACCCAT & \\
\hline GG_Rev_GGT & attGCTCTTCCACCACCGACTCGGTGCCACT & \\
\hline GG_For_GGT & attGCTCTTCAGGTTTTTTCCCCAGTTGCAAAAGTTGAC & \\
\hline GG_Rev_TTC & attGCTCTTCGGAAAAAACCACCGACTCGGTGCCACT & \\
\hline GG_For_TTC & attGCTCTTCATTCCCCAGTTGCAAAAGTTGAC & \\
\hline GG_Rev_E & attGCTCTTCAAAGCACCACCGACTCGGTGCCACT & \\
\hline \multicolumn{3}{|c|}{ Primers for directed mutagenesis for the removal of Sapl in LEU2 } \\
\hline RemSapl_Leu2_For & cgccgatatcggaggTtcttcctccacctc & \\
\hline RemSapl_Leu2_Rev & cctccgatatcggcggtagt & $p<\delta<2$ \\
\hline \multicolumn{3}{|c|}{ Oligonucleotides for the removal of Cas9 cassettes in generic plasmids } \\
\hline PL_For & cgcgtggatccggtaccactagtgtac & \multirow{2}{*}{ pCg57 and pCg58 } \\
\hline PL_Rev & actagtggtaccggatcca & \\
\hline \multicolumn{3}{|c|}{ Primers for directed mutagenesis for the construction of the multiplex backbone plasmids } \\
\hline Muta_Sapl_GG_For & AGCATTAGCTCTTCGCTTTTTTTGTTTITTATGTCTCCTAGGC & \multirow{2}{*}{ pg7, pg8, pCg57, pCg58 } \\
\hline Muta_Sapl_GGBB_rev & CGAAGAGCTAATGCTCTTCCTCAAGGGCATCGGTCGACA & \\
\hline \multicolumn{3}{|c|}{ Primer for sequencing verification of the sgRNA constructs } \\
\hline Seq_guide & TATGAATCTGCACGGTGTGC & pCg58, pg7, pCg69, or pg10 \\
\hline \multicolumn{3}{|c|}{ Primers for Yarrowia lipolytica loci sequencing } \\
\hline $\begin{array}{l}\text { POX1_For } \\
\text { POX1_Rev }\end{array}$ & $\begin{array}{l}\text { CTGCTCTGTCGCCTTATCTG } \\
\text { GGACAGAAGTTGCTCTCTGG }\end{array}$ & POX1 \\
\hline $\begin{array}{l}\text { POX2_For } \\
\text { POX2_Rev }\end{array}$ & $\begin{array}{l}\text { CCAAGCTGTCTCTGTTTGTG } \\
\text { GTGAGAGATCGGCCATTGAG }\end{array}$ & POX2 \\
\hline $\begin{array}{l}\text { POX3_For } \\
\text { POX3_Rev }\end{array}$ & $\begin{array}{l}\text { GCCACTGGAAGAAGGACACA } \\
\text { CTCAGAGTAGCCTGCTCAAG }\end{array}$ & POX3 \\
\hline $\begin{array}{l}\text { POX4_For } \\
\text { POX4_Rev }\end{array}$ & $\begin{array}{l}\text { TCCAGCTGTCCAAGTCCATC } \\
\text { TTCCAGGGATCGTCCGTTTC }\end{array}$ & POX4 \\
\hline $\begin{array}{l}\text { POX5_For } \\
\text { POX5_Rev }\end{array}$ & $\begin{array}{l}\text { ACAAGCTGTTGAGCGAATCG } \\
\text { GTGAGAGGCTTGTTGTCAAC }\end{array}$ & POX5 \\
\hline $\begin{array}{l}\text { POX6_For } \\
\text { POX6_Rev }\end{array}$ & $\begin{array}{l}\text { AGGAGACCAAGCTGATCGAC } \\
\text { GAGTGATGTAGCCCTCTCGG }\end{array}$ & POX6 \\
\hline $\begin{array}{l}\text { SPS191_dwst } \\
\text { SPS191_upst }\end{array}$ & $\begin{array}{l}\text { GGCCGTTACAGTAATGGAAC } \\
\text { ACAGCGTTCACGATAAGGAC }\end{array}$ & SPS191 \\
\hline $\begin{array}{l}\text { SPS192_dwst } \\
\text { SPS192_upst }\end{array}$ & $\begin{array}{l}\text { TACTGCCAGTGGGTTATTGC } \\
\text { GTCTCATTCTACCGAGGTAC }\end{array}$ & SPS192 \\
\hline $\begin{array}{l}\text { SPS193_upst } \\
\text { SPS193_Rev }\end{array}$ & $\begin{array}{l}\text { GACCAGCGTAGAGTAGCAGT } \\
\text { ACAACTGCGCTTAGAGCTTG }\end{array}$ & SPS193 \\
\hline
\end{tabular}




\begin{tabular}{|c|c|c|}
\hline \multicolumn{3}{|c|}{ Primers for the amplification of LEU2 marker } \\
\hline $\begin{array}{l}\text { tLeu2_Mlul_Rev } \\
\text { pLeu2_AatIl_For }\end{array}$ & $\begin{array}{l}\text { CATAAGGGAGACGCGTACCACTGTCCTCCACTA } \\
\text { GAAAAGTGCCACCTGACGTCTGAATTCCGCCTGAGTCA }\end{array}$ & JMP62-LEU2-ex-pTEF-dsRedStar² \\
\hline \multicolumn{3}{|c|}{ Primers for the amplification of URA3 marker } \\
\hline $\begin{array}{l}\text { tUra3_Mlul_Rev } \\
\text { pUra3_Aatll_For }\end{array}$ & $\begin{array}{l}\text { CATAAGGGAGACGCGTCCGAGAAACACAACAACATG } \\
\text { GAAAAGTGCCACCTGACGTCGAAGTTATACGAATTCGCG }\end{array}$ & JMP62-URA3-ex-pTEF-dsRedStar² \\
\hline \multicolumn{2}{|c|}{ Oligonucleotides used for target insertion of sgRNA in pg7 and pCg58 by Golden Gate reaction } & Target \\
\hline $\begin{array}{l}\text { gPOX1-5_ForG } \\
\text { gPOX1-5_Rev }\end{array}$ & $\begin{array}{l}\text { GCAgagtgccgacaggcctgtgg } \\
\text { AACccacaggcctgtcggcactc }\end{array}$ & POX1 +POX5 \\
\hline $\begin{array}{l}\text { gPOX2-3_ForG } \\
\text { gPOX2-3_Rev }\end{array}$ & $\begin{array}{l}\text { GCAgttggagccatggcccagct } \\
\text { AACagctgggccatggctccaac }\end{array}$ & $\mathrm{POX} 2+\mathrm{POX} 3$ \\
\hline $\begin{array}{l}\text { g2POX2-3_ForG } \\
\text { g2POX2-3_Rev }\end{array}$ & $\begin{array}{l}\text { GCAggccaggcctacgccgact } \\
\text { AACagtcggcgtaggcctggcc }\end{array}$ & $\mathrm{POX} 2+\mathrm{POX} 3$ \\
\hline $\begin{array}{l}\text { gPOX4-6_ForG } \\
\text { gPOX4-6_Rev }\end{array}$ & $\begin{array}{l}\text { GCAgacaggcgtgtggaggcca } \\
\text { AACtggcctccacacgcctgtc }\end{array}$ & POX4 + POX6 \\
\hline $\begin{array}{l}\text { SPS191g1_ForG } \\
\text { SPS191g1_Rev }\end{array}$ & $\begin{array}{l}\text { GCAtctgctggtagcatggtgta } \\
\text { AACtacaccatgctaccagcaga }\end{array}$ & SPS191 \\
\hline $\begin{array}{l}\text { SPS191g2_ForG } \\
\text { SPS191g2_Rev }\end{array}$ & $\begin{array}{l}\text { GCAaagcagtcgatccatacctt } \\
\text { AACaaggtatggatcgactgctt }\end{array}$ & SPS191 \\
\hline $\begin{array}{l}\text { SPS192g1_ForG } \\
\text { SPS192g1_Rev }\end{array}$ & $\begin{array}{l}\text { GCAagtgacacaaaaggccgcgg } \\
\text { AACccgcggccttttgtgtcact }\end{array}$ & SPS192 \\
\hline $\begin{array}{l}\text { SPS192g2_ForG } \\
\text { SPS192g2_Rev }\end{array}$ & $\begin{array}{l}\text { GCAgtggttccaaatctctgcac } \\
\text { AACgtgcagagatttggaaccac }\end{array}$ & SPS192 \\
\hline $\begin{array}{l}\text { SPS193g1_ForG } \\
\text { SPS193g1_Rev }\end{array}$ & $\begin{array}{l}\text { GCAagacagtgtgttgggcattg } \\
\text { AACcaatgcccaacacactgtct }\end{array}$ & SPS193 \\
\hline $\begin{array}{l}\text { SPS193g2_ForG } \\
\text { SPS193g2_Rev }\end{array}$ & $\begin{array}{l}\text { GCActgatccccgcccagcgatg } \\
\text { AACcatcgctgggcggggatcag }\end{array}$ & SPS193 \\
\hline $\begin{array}{l}\text { gSPS191/192_ForG } \\
\text { gSPS191/192_Rev }\end{array}$ & $\begin{array}{l}\text { GCAcaaatggtgcctgctcctc } \\
\text { AACgaggagcaggcaccatttg }\end{array}$ & SPS191 + SPS192 \\
\hline
\end{tabular}

\begin{tabular}{|c|c|}
\hline \multicolumn{2}{|r|}{ 90bp oligonucleotides for HDR on POX genes } \\
\hline POX1-stop & $\mathrm{c}^{*} \mathrm{c}^{*} \mathrm{a}^{*}$ cgtggggatgtgccaaaatcattgatgagtgccgaTaggcctgAggTAAcatggatactctggatataacggatttggccaggg ${ }^{*} \mathrm{c}^{*} \mathrm{a}$ \\
\hline POX4-stop & $\mathrm{g}^{*} \mathrm{~g}^{*} \mathrm{c}^{*}$ ttgtgctgacatcattgacaaggcccgacaggcgtgatgaggccacTAaactctgcctacaacggctttggacaggccttccag* ${ }^{*} \mathrm{a}^{*} \mathrm{c}$ \\
\hline POX5-stop & c* c*a*cctgggctgccgccaagatcattgacgagtgccgaTaggcctgAggtAAcatggctactccggctacaacggtttcggtcaggc*t*t*a \\
\hline POX6-stop & 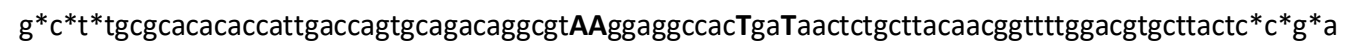 \\
\hline
\end{tabular}


Tables S2 Sequence identity inside $Y$. lipolytica gene families and CRISPR/Cas9 targets

Table S2.1 POX family-Nucleotide identity matrix of POX genes

\begin{tabular}{cccccc}
\hline Gene & POX6 & POX4 & POX1 & POX2 & POX3 \\
\hline POX6-YALIOE06567g & 100 & & & & \\
POX4-YALIOE27654g & 62,68 & 100 & & & \\
POX1-YALIOE32835g & 61,36 & 60,77 & 100 & & \\
POX2-YALIOF10857g & 66,55 & 68,24 & 66,65 & 100 & \\
POX3-YALIOD24750g & 65,7 & 65,46 & 68,97 & 72,22 & 100 \\
POX5-YALIOC23859g & 64,36 & 63,23 & 68,12 & 68,46 & 73,22 \\
\hline
\end{tabular}

Genes were aligned using Clustal W (http://www.genome.jp/tools-bin/clustalw)

Table S2.2 Identical sequences next to a NGG PAM in POX genes

\begin{tabular}{ccccc}
\hline Targeted gene & Guide sequences & \# bases & sgRNA designation \\
\cline { 1 - 4 } POX1 + POX5 & GAGTGCCGACAGGCCTGTGG & 20 & POX1/5 \\
POX2 + POX3 & GTTGGAGCCATGGCCCAGCT & 20 & POX2/3a \\
POX2 + POX3 & GGCCAGGCCTACGCCGACT & 19 & POX2/3b \\
POX 4+ POX6 & GACAGGCGTGTGGAGGCCA & 19 & POX4/6 \\
\hline
\end{tabular}


Table S2.3 SPS19 family-Nucleotide identity matrix of SPS19 genes

\begin{tabular}{ccc}
\hline Gene & SPS 191 & SPS 192 \\
\hline SPS191-YALIOD1694g & 100 & \\
SPS192-YALIOE14322g & 60.11 & 100 \\
SPS193-YALIOC03003g & 59.92 & 69.48 \\
\hline
\end{tabular}

Genes were aligned using Clustal W (http://www.genome.jp/tools-bin/clustalw)

Table S2.4 Target sequences in SPS19 genes

\begin{tabular}{cccc}
\hline Targeted gene & Guide sequences & \# bases & sgRNA designation \\
SPS191 & TCTGCTGGTAGCATGGTGTA & 20 & SPS1a \\
SPS191 & AAGCAGTCGATCCATACCTT & 20 & SPS1b \\
SPS192 & AGTGACACAAAAGGCCGCGG & 20 & SPS2a \\
SPS192 & GTGGTTCCAAATCTCTGCAC & 20 & SPS2b \\
SPS193 & AGACAGTGTGTTGGGCATTG & 20 & SPS3a \\
SPS193 & CTGATCCCCGCCCAGCGATG & 20 & SPS3b \\
SPS191 + SPS192 & CAAATGGTGCCTGCTCCTC & 19 & SPSm \\
\hline
\end{tabular}


Table S3 Genetic events (POX)

Table S3.1 Sequencing of double mutants (POX4/POX6)

\begin{tabular}{|c|c|c|c|c|}
\hline \multirow{2}{*}{$\begin{array}{l}\text { Locus } \\
\text { Colony }\end{array}$} & \multicolumn{2}{|c|}{ POX4 } & \multicolumn{2}{|c|}{ POX6 } \\
\hline & Genotype & Colony diversity & Genotype & Colony diversity \\
\hline P15_9A & del - 13 bp & unique & del - 11 bp & unique \\
\hline P15_9B & del - 1 bp & unique & del - 1 bp & unique \\
\hline P15_9C & del - 8 bp & unique & ins $G$ & unique \\
\hline P15_9D & del - 7 bp & unique & ins 147 bp (repeats) & unique \\
\hline P15_9E & del - 1 bp & unique & del - 1 bp & unique \\
\hline P15_9F & ins $G$ & unique & del - 14 bp & unique \\
\hline P15_9G & del $-7 b p+$ ins $2 b p$ & unique & del - 1 bp & unique \\
\hline P15_9H & del - 1 bp & Predominant phenotype & del - 1 bp & unique \\
\hline P15_10A & del - $13 b p$ & Predominant phenotype & del - 1 bp & unique \\
\hline P15_10C & del - 1 bp & unique & del - 1 bp & unique \\
\hline P15_10D & del - 1 bp & unique & del - 9 bp & unique \\
\hline P15_10E & del $-12 b p$ & unique & del - 33 bp & unique \\
\hline P15_10G & WT & unique & WT & unique \\
\hline P15_11A & del - 17 bp & unique & del - 1 bp & unique \\
\hline P15_11B & del - 16 bp & unique & del - 7 bp & unique \\
\hline P15_11C & WT & unique & WT & unique \\
\hline P15_11D & WT & unique & WT & unique \\
\hline P15_11E & del -3bp & unique & del - 1 bp & unique \\
\hline P15_11F & del -18bp & unique & del - 12 bp & Predominant phenotype \\
\hline P15_11G & del - 1 bp & unique & ins $G$ & unique \\
\hline P15_11H & del - 1 bp & Predominant phenotype & del - 1 bp & unique \\
\hline & \multicolumn{2}{|c|}{ chr E ex-POX 6 (POX6_For/POX4_For) } & \multicolumn{2}{|c|}{ chr E ex-POX 4 (POX6_Rev/POX4_rev) } \\
\hline P15_10B & Junction POX4->POX6 & unique & Junction POX4->POX6 & unique \\
\hline P15_10F & Junction POX4->POX6 & unique & Junction POX4->POX6 & unique \\
\hline P15_10H & Junction POX4->POX6 & unique & Junction POX4->POX6 & unique \\
\hline
\end{tabular}


Table S3.2 Sequencing of double mutants (POX1/POX5)

\begin{tabular}{|c|c|c|c|c|}
\hline \multirow{2}{*}{$\begin{array}{l}\text { Locus } \\
\text { Colony }\end{array}$} & \multicolumn{2}{|c|}{ POX1 } & \multicolumn{2}{|c|}{ POX5 } \\
\hline & Genotype & Colony diversity & Genotype & Colony diversity \\
\hline P46_4A & del - 2 bp & unique & del - 2 bp & unique \\
\hline P46_4B & del - 2 bp & unique & del - 2 bp & unique \\
\hline P46_4C & del - 1 bp & unique & del - 453 bp & unique \\
\hline P46_4D & del - 1 bp & unique & del - 2 bp & unique \\
\hline P46_4E & del - 1 bp & unique & del - 1 bp & unique \\
\hline P46_4F & del - $11 b p$ & unique & del - 2 bp & unique \\
\hline P46_4G & del - 2 bp & unique & del - 1 bp & unique \\
\hline P46_4H & del - 1 bp & unique & del - 2 bp & unique \\
\hline P46_5A & del - 2 bp & unique & undetermined & $\operatorname{mix}$ \\
\hline P46_5B & del - 1 bp & unique & del - 2 bp & unique \\
\hline P46_5C & ins 54 bp (salmo salar) & unique & del - 1 bp & unique \\
\hline P46_5D & del - 1 bp & unique & del - 2 bp & unique \\
\hline P46_5E & del - 1 bp & unique & del - 37 bp & Predominant phenotype \\
\hline P46_5F & del - 2 bp & unique & del - 2 bp & unique \\
\hline P46_5H & del - $2 b p$ & unique & del - 1 bp & unique \\
\hline P46_6A & del - 2 bp & unique & \multicolumn{2}{|c|}{ No PCR amplification } \\
\hline P46_6B & del - 1 bp & unique & ins TG & unique \\
\hline P46_6C & del - 2 bp & unique & del - 2 bp & unique \\
\hline P46_6D & del - 2 bp & unique & del - 2 bp & unique \\
\hline P46_6E & del - 1 bp & unique & del - 1 bp & unique \\
\hline P46_6F & ins 51 bp (salmo salar) & unique & del - 2 bp & unique \\
\hline P46_6G & del - 1 bp & unique & del - 1 bp & unique \\
\hline P46_6H & del - 1 bp & unique & del - 2 bp & unique \\
\hline & \multicolumn{2}{|c|}{ chr E ex-POX 1 (POX1_For/POX5_For) } & \multicolumn{2}{|c|}{ chr C ex-POX 5 (POX1_Rev/POX5_Rev) } \\
\hline P46_5G & Junction POX1->POX5 & unique & Junction POX5->POX1 & unique \\
\hline
\end{tabular}


Table S3.3 Sequencing of double mutants (POX2/POX3)

\begin{tabular}{|c|c|c|c|c|}
\hline \multirow[b]{2}{*}{ Colony } & \multicolumn{2}{|l|}{ POX2 } & \multicolumn{2}{|l|}{ POX3 } \\
\hline & Genotype & Colony diversity & Genotype & Colony diversity \\
\hline P23_A1 & Excision 846 bp & unique & Excision 846 bp & unique \\
\hline P23_C1 & Excision 846 bp & unique & Flip 846 bp & unique \\
\hline P23_E1 & Excision $846 \mathrm{bp}+$ ins $\mathrm{T}$ & unique & Excision $846 \mathrm{bp}+$ ins $\mathrm{T}$ & unique \\
\hline P23_F1 & Excision 846 bp & unique & Excision 846 bp & unique \\
\hline P23_G1 & Excision 846 bp & unique & Excision 846 bp & unique \\
\hline P23_A2 & Flip 846 pb + ins T POX2a & unique & Excision 846 bp & unique \\
\hline P23_C2 & Excision 846 bp & unique & Flip 846 bp + del -3/4 bp POX3b & $\begin{array}{c}\text { unique at } \\
\text { POX3a, mix at } \\
\text { POX3b }\end{array}$ \\
\hline P23_E2 & Excision 846 bp & unique & Excision & unique \\
\hline P23_F2 & Flip 846 bp + del 2 bp POX2b & unique & Flip 846 bp + del 3bp POX3b & unique \\
\hline P23_G2 & Excision 846 bp & unique & Excision or flip 846 bp & $\operatorname{mix}$ \\
\hline \multirow[t]{2}{*}{ P23_H2 } & $\begin{array}{c}\text { del }-1 \text { bp at POX2a } \\
\text { various indels at POX2b }\end{array}$ & $\begin{array}{l}\text { mix at both DSB } \\
\text { sites }\end{array}$ & Excision & unique \\
\hline & Chr D ex-POX3 (POX2_For & OX3_Rev) & \multicolumn{2}{|c|}{ ChrF ex-POX2 (POX3_For/POX2_Rev) } \\
\hline P23_D1 & Junction POX2a->POX3b + ins T & unique & Junction POX3a->POX2 b & unique \\
\hline P23_H1 & Amplification failed & & Junction POX3a->POX2b & unique \\
\hline P23_B2 & Junction POX2a->POX3b & unique & $\begin{array}{c}\text { Junction POX3b->POX2a } \\
\text { various indels POX } 2 \mathrm{~b}\end{array}$ & $\operatorname{mix}$ \\
\hline P23_D2 & Junction POX2a->POX3b & unique & $\begin{array}{l}\text { Junction POX3a-> flip } 846 \text { bp POX3b } \\
->\text { POX } 2 b+\text { del } 4 \text { bp at POX2a }\end{array}$ & unique \\
\hline
\end{tabular}


Table S3.4 Sequencing of quadruple mutants (POX1, POX4, POX5, POX6) without oligomers

\begin{tabular}{|c|c|c|c|c|}
\hline Colony & POX1 & POX4 & POX5 & POX6 \\
\hline \multirow{2}{*}{ P1456_A1 } & WT & WT & WT & WT \\
\hline & unique & unique & unique & unique \\
\hline \multirow{2}{*}{ P1456_A2 } & WT & WT & WT & WT \\
\hline & unique & unique & unique & unique \\
\hline \multirow{2}{*}{ P1456_A3 } & WT & WT & WT & WT \\
\hline & unique & unique & unique & unique \\
\hline \multirow{2}{*}{ P1456_A4 } & & del $-1 b p$ & del $-1 b p$ & del $-1 b p$ \\
\hline & unique & unique & unique & unique \\
\hline P1456_A5 & $\begin{array}{c}\text { del }-9 b p \text {, ins+3 bp } \\
\text { unique }\end{array}$ & cf. translocation table S3.6 & $\begin{array}{l}\text { del -16 bp } \\
\text { unique }\end{array}$ & cf. translocation table S3.6 \\
\hline \multirow{2}{*}{ P1456_A6 } & del $-2 b p$ & del -2 bp & del $-2 b p$ & del $-9 b p$ \\
\hline & unique & unique & unique & unique \\
\hline \multirow{2}{*}{ P1456_A7 } & WT & WT & WT & WT \\
\hline & unique & unique & unique & unique \\
\hline \multirow{2}{*}{ P1456_A8 } & WT & & WT & WT \\
\hline & unique & unique & unique & unique \\
\hline \multirow{2}{*}{ P1456_A9 } & WT & WT & WT & WT \\
\hline & unique & unique & unique & unique \\
\hline P1456_A10 & $\begin{array}{l}\text { cf. translocation table } \\
\text { S3.6 }\end{array}$ & $\begin{array}{l}\text { ins A } \\
\text { unique }\end{array}$ & \multicolumn{2}{|c|}{ cf. translocation table S3.6 } \\
\hline \multirow{2}{*}{ P1456_A11 } & WT & del $-1 b p$ & del $-2 b p$ & ins 108 bp (Cyprinus carpio) \\
\hline & unique & unique & unique & unique \\
\hline P1456_A12 & \multicolumn{4}{|c|}{ cf. translocation table S3.6 } \\
\hline \multirow{2}{*}{ P1456_B1 } & WT & WT & WT & del $-1 b p$ \\
\hline & unique & Predominant & unique & $\operatorname{mix}$ \\
\hline \multirow{2}{*}{ P1456_B2 } & del $-1 b p$ & del -1 bp & del $-2 b p$ & del $-1 b p$ \\
\hline & unique & unique & unique & unique \\
\hline \multirow{2}{*}{ P1456_B3 } & WT & WT & WT & WT \\
\hline & unique & unique & unique & unique \\
\hline P1456_B4 & $\begin{array}{c}\text { del -1 bp } \\
\text { unique }\end{array}$ & cf. translocation table S3.6 & $\begin{array}{c}\text { del -4 bp } \\
\text { unique }\end{array}$ & cf. translocation table S3.6 \\
\hline \multirow{2}{*}{ P1456_B5 } & WT & WT & WT & WT \\
\hline & unique & unique & unique & unique \\
\hline P1456_B6 & $\begin{array}{c}\text { cf. translocation table } \\
\text { S3.6 }\end{array}$ & $\begin{array}{l}\text { del -1 bp } \\
\text { unique }\end{array}$ & \multicolumn{2}{|c|}{ cf. translocation table S3.6 } \\
\hline \multirow{2}{*}{ P1456_B7 } & del -2bp & del $-1 b p$ & del $-2 b p$ & del $-1 b p$ \\
\hline & unique & unique & unique & unique \\
\hline \multirow{2}{*}{ P1456_B8 } & WT & WT & WT & WT \\
\hline & unique & unique & unique & unique \\
\hline \multirow{2}{*}{ P1456_B9 } & WT & WT & WT & WT \\
\hline & unique & unique & unique & unique \\
\hline \multirow{2}{*}{ P1456_B10 } & WT & WT & WT & WT \\
\hline & unique & unique & unique & unique \\
\hline \multirow{2}{*}{ P1456_B11 } & WT & WT & WT & WT \\
\hline & unique & unique & unique & unique \\
\hline
\end{tabular}


Table S3.5 Sequencing of quadruple mutants (POX1, POX4, POX5, POX6) with oligomers

\begin{tabular}{|c|c|c|c|c|}
\hline Colony & POX1 & POX4 & POX5 & POX6 \\
\hline \multirow{2}{*}{ P1456_E1 } & WT & WT & WT & WT \\
\hline & unique & unique & unique & unique \\
\hline \multirow{2}{*}{ P1456_E2 } & \multirow{2}{*}{ cf. translocation table S3.6 } & & HDR & of translocation table $\$ 36$ \\
\hline & & unique & unique & -1. (r) \\
\hline \multirow{2}{*}{ P1456_E3 } & WT & WT & WT & WT \\
\hline & unique & unique & unique & unique \\
\hline P1456_E4 & \multicolumn{4}{|c|}{ cf. translocation table S3.6 } \\
\hline \multirow{2}{*}{ P1456_E5 } & WT & WT & WT & WT \\
\hline & unique & unique & unique & unique \\
\hline \multirow{2}{*}{ P1456_E6 } & HDR & HDR & HDR (partial)* & ins $\mathrm{G}$ \\
\hline & unique & unique & unique & unique \\
\hline \multirow{2}{*}{ P1456_E7 } & WT & WT & WT & WT \\
\hline & unique & unique & unique & unique \\
\hline \multirow{2}{*}{ P1456_E8 } & HDR & HDR & HDR & HDR \\
\hline & unique & unique & unique & unique \\
\hline \multirow{2}{*}{ P1456_E9 } & WT & WT & WT & WT \\
\hline & unique & unique & unique & unique \\
\hline \multirow{2}{*}{ P1456_E10 } & WT & WT & WT & WT \\
\hline & unique & unique & unique & unique \\
\hline \multirow{2}{*}{ P1456_E11 } & del $-1 b p$ & del $-20 b p$ & del $-1 b p$ & del $-1 b p$ \\
\hline & unique & unique & unique & unique \\
\hline \multirow{2}{*}{ P1456_E12 } & WT & WT & WT & WT \\
\hline & unique & unique & unique & unique \\
\hline \multirow{2}{*}{ P1456_F1 } & WT & WT & WT & WT \\
\hline & unique & unique & unique & unique \\
\hline \multirow{2}{*}{ P1456_F2 } & WT & WT & WT & WT \\
\hline & unique & unique & unique & unique \\
\hline \multirow{2}{*}{ P1456_F3 } & HDR & HDR & HDR (partial)* & oligomer insertion (+78 bp) \\
\hline & unique & unique & unique & unique \\
\hline \multirow{2}{*}{ P1456_F4 } & WT & HDR & WT & HDR \\
\hline & unique & unique & $\operatorname{mix}$ & unique \\
\hline \multirow{2}{*}{ P1456_F5 } & WT & HDR & HDR & WT \\
\hline & $\operatorname{mix}$ & unique & unique & $\operatorname{mix}$ \\
\hline \multirow{2}{*}{ P1456_F6 } & WT & WT & WT & WT \\
\hline & unique & unique & unique & unique \\
\hline \multirow{2}{*}{ P1456_F7 } & HDR & del $-1 b p$ & HDR & oligomer insertion (+178 bp) + HDR \\
\hline & unique & unique & unique & unique \\
\hline \multirow{2}{*}{ P1456_F8 } & WT & WT & WT & WT \\
\hline & unique & unique & unique & unique \\
\hline \multirow{2}{*}{ P1456_F9 } & HDR & HDR & del - $2 b p$ & del $-1 b p$ \\
\hline & unique & unique & unique & unique \\
\hline \multirow{2}{*}{ P1456_F10 } & del $-2 b p$ & del $-1 b p$ & WT & oligomer insertion (+90 bp) + HDR \\
\hline & unique & unique & $\operatorname{mix}$ & unique \\
\hline
\end{tabular}

$\left({ }^{*}\right)$ : partial HDR occurs only on one side of the DSB, when the other side remains WT 
Table S3.6 Summary of detected chromosomal translocations in quadruple mutants (POX1, POX4, POX5, POX6)

When Colony PCR failed with the standard forward/reverse primer combination, the combinations were modified to screen for translocations. Positive PCRs where sequenced.

\begin{tabular}{|c|c|c|c|}
\hline P1456_A5 & $\begin{array}{c}\text { POX4_Rev/POX6_Rev: } \\
\text { junction POX4-POX6 } \\
\text { unique }\end{array}$ & $\begin{array}{c}\text { POX4_For/POX6_For } \\
\text { junction POX4-POX6 } \\
\text { unique }\end{array}$ & \\
\hline P1456_A10 & $\begin{array}{c}\text { POX1_Rev/POX6_For } \\
\text { junction POX6->POX1 } \\
\text { unique }\end{array}$ & $\begin{array}{c}\text { PO5_Rev/POX6_Rev } \\
\text { junction POX5- POX6 + ins } 73 \text { bp (unk) } \\
\text { unique }\end{array}$ & \\
\hline P1456_A12 & $\begin{array}{c}\text { PO5_Rev/POX6_Rev } \\
\text { junction POX5->POX6 } \\
\text { unique }\end{array}$ & $\begin{array}{cc}\text { POX1_Rev/POX4_For } & \text { POX4_Rev/POX1_For } \\
\text { junction POX4-> POX1 } & \text { junction POX1-> POX4 } \\
\text { del }-11 \text { bp }+52 \text { bp (S. salar) } & \text { del }-4 b p \\
\text { unique } & \text { unique }\end{array}$ & $\begin{array}{l}\text { POX5_For/POX6_For } \\
\text { junction POX5- POX6 } \\
+ \text { ins } 339 \text { bp (S. salar) } \\
\text { unique }\end{array}$ \\
\hline P1456_B4 & $\begin{array}{c}\text { POX4_For/POX6_For } \\
\text { junction POX4-POX6 } \\
\text { unique }\end{array}$ & $\begin{array}{c}\text { POX4_Rev/POX6_Rev } \\
\text { junction POX4-POX6 } \\
\text { unique }\end{array}$ & \\
\hline P1456_B6 & $\begin{array}{c}\text { POX1_Rev/POX6_For } \\
\text { junction POX6-> POX1 } \\
\text { del }-148 \text { bp }+\mathrm{T} \\
\text { Unique }\end{array}$ & & \\
\hline P1456_E2 & $\begin{array}{c}\text { POX1_For/POX6_Rev } \\
\text { junction POX1-> POX6 } \\
\text { unique }\end{array}$ & $\begin{array}{c}\text { POX1_Rev/POX6_For } \\
\text { junction POX6-> POX1 } \\
\text { unique }\end{array}$ & \\
\hline P1456_E4 & $\begin{array}{l}\text { POX4_Rev/POX5_For } \\
\text { mix of amplicons } \\
\text { mix }\end{array}$ & $\begin{array}{l}\text { POX4_For/POX5_Rev } \\
\text { junction POX4->POX5 } \\
\text { del }-1 \text { bp } \\
\text { unique }\end{array}$ & $\begin{array}{c}\text { POX1_For/POX6_Rev } \\
\text { junction POX1-> POX } 6 \\
\text { del }-5 \text { bp + ins } 53 \text { bp (S. salar) } \\
\text { unique }\end{array}$ \\
\hline
\end{tabular}




\section{Table S4 Genetic events (SPS)}

Table S4.1-Different susceptibility to CRISPR/Cas9 between SPS191 and SPS192 locus Clones transformed with pCg58-SPSm (plasmid with only one sgRNA targeting both SPS191 and SPS192) were analysed by colony PCR at SPS191, SPS192, and SPS193 locus (see section SI-molecular biology for conditions). All Sanger sequencing chromatograms were processed with TIDE in order to quantify the proportion of each genotype within the colony. PCR of the SPS193 locus of the 15 hereby clones, as well as 8 other randomly picked colonies were all identical to WT. (SPS193 has an almost identical sequence with a single mismatch to SPSm).

\begin{tabular}{|c|c|c|c|c|c|c|c|c|}
\hline \multirow[b]{2}{*}{ Colony } & \multicolumn{4}{|c|}{ SPS191 } & \multicolumn{4}{|c|}{ SPS192 } \\
\hline & Major genotype & $\%$ TIDE & Secondary geno & $\%$ TIDE & Major genotype & $\%$ TIDE & Secondary genotype & $\%$ TIDE \\
\hline S12_11A & WT & 83,1 & del $-1 b p$ & 10,9 & del-10bp & 99,2 & N.A. & \\
\hline S12_11B & WT & 89,8 & del $-1 b p$ & 5,1 & del $-3 b p$ & 99,1 & N.A. & \\
\hline S12_11C & WT & 87,9 & del -1 bp & 7,0 & del-1bp & 98,9 & N.A. & \\
\hline S12_11D & del $-3 b p$ & 43,8 & WT & 23,7 & del $-1 b p$ & 99,0 & N.A. & \\
\hline S12_11E & WT & 89,0 & del -7 bp & 3,1 & del $-1 b p$ & 98,7 & N.A. & \\
\hline S12_11F & del $-8 b p$ & 79,5 & WT & 15,0 & del $-8 b p+$ ins $A A$ & 95,6 & del -1 bp & 3,8 \\
\hline S12_11G & WT & 90,1 & del $-1 b p$ & 2,8 & del $-1 b p$ & 99,1 & N.A. & \\
\hline $\mathrm{S} 12 \_11 \mathrm{H}$ & WT & 65,1 & del $-1 b p$ & 30,7 & del $-1 b p$ & 90,5 & del $-10 b p$ & 6,8 \\
\hline S12_12A & del -1 bp & 77,5 & WT & 18,3 & del $-1 b p$ & 46,6 & del $-8 b p$ & 36,7 \\
\hline S12_12B & WT & 91,1 & del -1 bp & 3,6 & del $-1 b p$ & 99,3 & N.A. & \\
\hline S12_12BC & WT & 64,9 & del $-10 b p$ & 16,5 & del $-1 b p$ & 92,2 & del $-3 b p$ & 6,9 \\
\hline S12_12D & WT & 84,1 & del $-1 b p$ & 8,4 & del $-1 b p$ & 98,9 & N.A. & \\
\hline S12_12E & WT & 84,3 & del $-1 b p$ & 7,0 & del $-1 b p$ & 76,2 & del $-25 b p$ & 17,9 \\
\hline S12_12F & WT & 66,7 & del -1 bp & 17,1 & del $-1 b p$ & 95,5 & WT & 3,2 \\
\hline S12_12G & WT & 71,6 & del -1 bp & 17,0 & del $-1 b p$ & 86,4 & del -8bp & 7,3 \\
\hline
\end{tabular}

Chromatogram traces were analysed with the TIDE online tool: https://tide.deskgen.com/ ${ }^{3}$. 
Table S4.2 Summary of sequencing results of colonies from mono-disruptions of SPS genes

\begin{tabular}{c|cc|cc|cc}
\hline & \multicolumn{2}{|c|}{ SPS191 } & \multicolumn{2}{c|}{ SPS192 } & \multicolumn{2}{c}{ SPS193 } \\
& SPS1a & SPS1b & SPS2a & SPS2b & SPS3a & SPS3b \\
\hline Excision & 8 & 8 & 8 & 8 & 12 & 12 \\
Flip & 1 & 1 & 8 & 8 & 3 & 3 \\
Indel & 5 & 5 & 0 & 0 & 1 & 1 \\
WT & 1 & 1 & 0 & 0 & 0 & 0 \\
\hline Total & 15 & 15 & 16 & 16 & 16 & 16 \\
\hline
\end{tabular}

Clones transformed simultaneously with either pCg58-SPS1a and pg10-SPS1b, or pCg58-SPS2a and pg10-SPS2b, or pCg58-SPS3a and pg10-SPS3b were analysed by colony PCR at SPS191, SPS192, and SPS193 locus (see section SI-molecular biology for conditions).

Table S4.3 Summary of sequencing results of colonies from double-disruptions of SPS genes

\begin{tabular}{|c|c|c|c|c|c|c|c|c|c|c|c|c|}
\hline & \multicolumn{4}{|c|}{ SPS192 + SPS193 } & \multicolumn{4}{|c|}{ SPS191 + SPS193 } & \multicolumn{4}{|c|}{ SPS191 + SPS192 } \\
\hline & \multicolumn{2}{|c|}{ SPS192 } & \multicolumn{2}{|c|}{ SPS193 } & \multicolumn{2}{|c|}{ SPS191 } & \multicolumn{2}{|c|}{ SPS193 } & \multicolumn{2}{|c|}{ SPS191 } & \multicolumn{2}{|c|}{ SPS192 } \\
\hline & SPS2a & SPS2b & SPS3a & SPS3b & SPS1a & SPS1b & SPS3a & SPS3b & SPSm & SPS1b & SPSm & SPS2b \\
\hline Excision & 4 & 4 & 10 & 10 & 8 & 8 & 10 & 10 & 8 & 8 & 8 & 8 \\
\hline Flip & 8 & 8 & 1 & 1 & 1 & 1 & 3 & 3 & 1 & 1 & 4 & 4 \\
\hline Indel & 1 & 1 & 2 & 2 & 4 & 4 & 1 & 1 & 0 & 2 & 1 & 0 \\
\hline DNA insertion & 0 & 0 & 1 & 1 & 0 & 0 & 0 & 0 & 0 & 2 & 0 & 1 \\
\hline DNA insertion + Excision & 1 & 1 & 0 & 0 & 0 & 0 & 0 & 0 & 0 & 0 & 0 & 0 \\
\hline DNA insertion + Flip & 0 & 0 & 0 & 0 & 1 & 1 & 0 & 0 & 0 & 0 & 0 & 0 \\
\hline Rearrangement & 1 & 1 & 1 & 1 & 2 & 2 & 2 & 2 & 0 & 0 & 0 & 0 \\
\hline WT & 1 & 1 & 1 & 1 & 0 & 0 & 0 & 0 & 7 & 3 & 3 & 3 \\
\hline Total & 16 & 16 & 16 & 16 & 16 & 16 & 16 & 16 & 16 & 16 & 16 & 16 \\
\hline
\end{tabular}

Clones transformed simultaneously with either pCg69-SPS192 and pg10-SPS193, or pCg69-SPS191 and pg10-SPS193, or pCg69-191/192 alone were analysed by colony PCR at SPS191, SPS192, and SPS193 locus

Table S4.4 Summary of sequencing results of colonies from triple disruptions of SPS genes

\begin{tabular}{l|cccccc}
\hline & \multicolumn{2}{|c}{ SPS191 } & \multicolumn{2}{c}{ SPS192 } & \multicolumn{2}{c}{ SPS193 } \\
& SPSm & SPS1b & SPSm & SPS2b & SPS3a & SPS3b \\
\hline Excision & 2 & 2 & 9 & 9 & 11 & 11 \\
Flip & 2 & 2 & 3 & 3 & 2 & 2 \\
Indel & 2 & 5 & 0 & 1 & 1 & 0 \\
DNA insertion & 1 & 2 & 0 & 0 & 0 & 0 \\
\hline DNA insertion + Excision & 3 & 3 & 0 & 0 & 2 & 2 \\
DNA insertion + Flip & 0 & 0 & 2 & 2 & 0 & 0 \\
\hline Rearrangement & 4 & 4 & 3 & 3 & 2 & 2 \\
WT & 10 & 6 & 7 & 6 & 6 & 7 \\
\hline & 24 & 24 & 24 & 24 & 24 & 24 \\
\hline
\end{tabular}

Clones transformed simultaneously with pCg69-191/192 and pg10-SPS193 were analysed by colony PCR at SPS191, SPS192, and SPS193 locus 


\section{Table S5 Summary table of all sequenced junctions}

The regions upstream of the gene are indicated by a box, the regions downstream of the gene is represented by an arrow, the chromosome to which they belong prior to translocation are colourcoded (blue: $\mathrm{Chr} C$, red: chr D, .green: chr E, purple: chr F), the POX genes regions are indicated by a single number corresponding to the POX gene number, the SPS gene regions by "SPS" followed by their last digit.

Table S5.1 List of fully characterised junctions resulting from single gene targeting Data aggregation for POX1, POX4, POX5, and POX6 targeting experiments

\begin{tabular}{|c|c|c|}
\hline & PCR & Sequenced junction \\
\hline $\begin{array}{lll} & 1 \\
& \end{array}$ & $\begin{array}{l}\text { POX1_For/ } \\
\text { POX4_Rev }\end{array}$ & 1 \\
\hline & $\begin{array}{l}\text { POX1_Rev/ } \\
\text { POX4_For }\end{array}$ & 1 \\
\hline \begin{tabular}{|l|l|}
5 & 1 \\
\end{tabular} & $\begin{array}{l}\text { POX1_For/ } \\
\text { POX5_For }\end{array}$ & 1 \\
\hline & $\begin{array}{l}\text { POX1_Rev/ } \\
\text { POX5_Rev }\end{array}$ & 1 \\
\hline & $\begin{array}{l}\text { POX1_For/ } \\
\text { POX6_Rev }\end{array}$ & 2 \\
\hline & $\begin{array}{l}\text { POX1_Rev/ } \\
\text { POX6_For }\end{array}$ & 3 \\
\hline & $\begin{array}{l}\text { POX4_For/ } \\
\text { POX5_Rev }\end{array}$ & 1 \\
\hline & $\begin{array}{l}\text { POX4_Rev/ } \\
\text { POX5_For }\end{array}$ & 1 \\
\hline & $\begin{array}{l}\text { POX4_For/ } \\
\text { POX6_For }\end{array}$ & 1 \\
\hline & $\begin{array}{l}\text { POX4_Rev/ } \\
\text { POX6_Rev }\end{array}$ & 5 \\
\hline 5 & $\begin{array}{l}\text { POX5_For/ } \\
\text { POX6_For }\end{array}$ & 1 \\
\hline & $\begin{array}{l}\text { POX5_For/ } \\
\text { POX6_For }\end{array}$ & 1 \\
\hline SPS2 SPS1 & $\begin{array}{l}\text { SPS191_upst/ } \\
\text { SPS192_dwst }\end{array}$ & 1 \\
\hline SPS1 SPS2 & $\begin{array}{l}\text { SPS192_upst/ } \\
\text { SPS191_dwst }\end{array}$ & 1 \\
\hline SPS3 SPS1 & $\begin{array}{l}\text { SPS191_upst/ } \\
\text { SPS193_upst }\end{array}$ & 2 \\
\hline
\end{tabular}


Table S5.2 List of fully characterised junctions resulting from double gene targeting Data aggregation for SPS 191, SPS192, 193, POX2 and POX3 targeting by excision strategy experiments.

\begin{tabular}{|c|c|c|}
\hline & PCR & Sequenced junction \\
\hline 322 & $\begin{array}{c}\text { POX2_For/ } \\
\text { POX3_Rev }\end{array}$ & 3 \\
\hline 3 2 & $\begin{array}{l}\text { POX2_Rev/ } \\
\text { POX3_For }\end{array}$ & 3 \\
\hline SPS2 SPS1 & $\begin{array}{l}\text { SPS191_upst/ } \\
\text { SPS192_dwst }\end{array}$ & 2 \\
\hline SPS1 SPS2 & $\begin{array}{l}\text { SPS192_upst/ } \\
\text { SPS191_dwst }\end{array}$ & 2 \\
\hline SPS3 SPS1 & $\begin{array}{l}\text { SPS191_upst/ } \\
\text { SPS193_upst }\end{array}$ & 3 \\
\hline SPS1 SPS3 & $\begin{array}{l}\text { SPS191_dwst/ } \\
\text { SPS193_dwst }\end{array}$ & 4 \\
\hline SPS3 SPS2 & $\begin{array}{l}\text { SPS192_upst/ } \\
\text { SPS19_upst }\end{array}$ & 2 \\
\hline SPS2 SPS3 & $\begin{array}{l}\text { SPS192_dwst/ } \\
\text { SPS193_dwst }\end{array}$ & 1 \\
\hline
\end{tabular}

\section{Table S6 Origin of large DNA fragment insertion in SPS disruptants in large} insertion events

When large insertion events were detected in the double disruptants ( 5 colonies out of the 48 analysed) and in the triple disruptants ( 8 out of 24 colonies), they were identified by comparison with the other SPS genes of $Y$. lipolytica, or by global alignment search using BLAST.

(https://blast.ncbi.nlm.nih.gov/Blast.cgi?PAGE TYPE=BlastSearch)

\begin{tabular}{c|ccc|cc|cc|cc}
\hline & \multicolumn{3}{|c|}{ Triple-excisions } & \multicolumn{4}{c}{ Double-excisions } \\
& SPS191 & SPS192 & SPS193 & SPS192 & SPS193 & SPS191 & SPS193 & SPS191 & SPS192 \\
\hline Other truncated SPS fragment & 4 & 1 & 2 & 0 & 0 & 1 & 0 & 2 & 1 \\
S. salar (DNA carrier) & 0 & 1 & 0 & 0 & 2 & 0 & 0 & 0 & 0 \\
Mitochondrion & 0 & 0 & 0 & 1 & 0 & 0 & 0 & 0 & 0 \\
\hline
\end{tabular}




\section{References}

(1) Borsenberger, V.; Onésime, D.; Lestrade, D.; Rigouin, C.; Neuvéglise, C.; Daboussi, F.; Bordes, F. Multiple Parameters Drive the Efficiency of CRISPR/Cas9-Induced Gene Modifications in Yarrowia Lipolytica. J. Mol. Biol. 2018. https://doi.org/10.1016/j.jmb.2018.08.024.

(2) Nicaud, J.-M.; Madzak, C.; van den Broek, P.; Gysler, C.; Duboc, P.; Niederberger, P.; Gaillardin, C. Protein Expression and Secretion in the Yeast Yarrowia Lipolytica. FEMS Yeast Res. 2002, 2 (3), 371-379. https://doi.org/10.1111/j.1567-1364.2002.tb00106.x.

(3) Brinkman, E. K.; Chen, T.; Amendola, M.; van Steensel, B. Easy Quantitative Assessment of Genome Editing by Sequence Trace Decomposition. Nucleic Acids Res. 2014, 42 (22), e168e168. https://doi.org/10.1093/nar/gku936. 